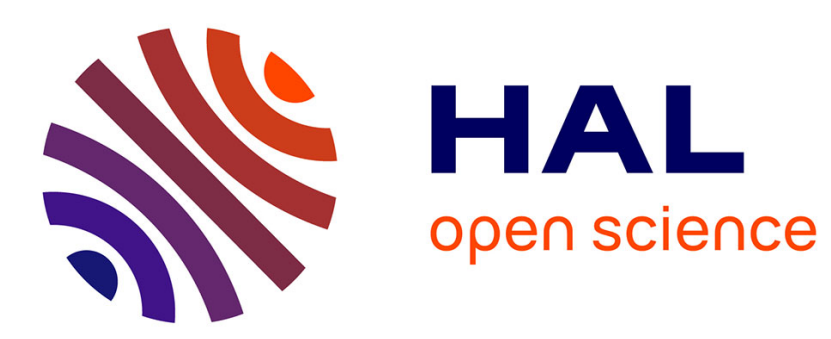

\title{
Cramér-Rao bound and statistical resolution limit investigation for near-field source localization
} Tao Bao, Mohammed Nabil El Korso, Habiba Hafdallah Ouslimani

\section{To cite this version:}

Tao Bao, Mohammed Nabil El Korso, Habiba Hafdallah Ouslimani. Cramér-Rao bound and statistical resolution limit investigation for near-field source localization. Digital Signal Processing, 2016, 48, pp.137-147. 10.1016/j.dsp.2015.09.019 . hal-01421517

\section{HAL Id: hal-01421517 \\ https://hal.parisnanterre.fr/hal-01421517}

Submitted on 4 Dec 2017

HAL is a multi-disciplinary open access archive for the deposit and dissemination of scientific research documents, whether they are published or not. The documents may come from teaching and research institutions in France or abroad, or from public or private research centers.
L'archive ouverte pluridisciplinaire HAL, est destinée au dépôt et à la diffusion de documents scientifiques de niveau recherche, publiés ou non, émanant des établissements d'enseignement et de recherche français ou étrangers, des laboratoires publics ou privés. 


\title{
Cramér-Rao bound and statistical resolution limit investigation for near-field source localization
}

\author{
Tao Bao ${ }^{a}$, Mohammed Nabil El Korso ${ }^{\text {b, }}$, Habiba Hafdallah Ouslimani ${ }^{\mathrm{b}}$ \\ a Department of Electronics Engineering, Northwestern Polytechnical University, Xi'an 710129, China \\ b Paris Ouest University, LEME EA4416, 50 rue de Sèvres, 92410 Ville d'Avray, France
}

\section{Introduction}

There has been tremendous interest in the investigation of array signal processing during the last three decades due to its importance in radar, sonar, seismology and communication systems [2-9]. One can classify source localization, in the context of array processing, into the so-called far-field and near-field cases. The context of far-field sources assumes planar wavefronts of the propagating waves as they reach the array. Nevertheless, when the sources are located in the so-called near-field region, this assumption is no longer valid. More precisely, the source wavefronts must be assumed spherical, which requires both the range and the direction-of-arrival (DOA) parameter estimation to carry out the localization procedure [10-12]. One can find several estimation algorithms adapted for the near-field source localization [13-17] and some related performance analysis in order to quantify the estimator performance in terms of the mean square error (MSE) [18-22]. However, to fully characterize estimator performance, one has, additionally, to perform the resolvability analysis of two closely spaced sources. Recently, some works considered both far field and near field scenario. In [23-25] the authors studied, respectively, the asymptotic resolvability of far-field, deterministic, discrete and stochastic source signals, whereas, in [26] the authors focused on

$$
\text { [1]. }
$$

This work was partially presented during the International Conference SAM-12

* Corresponding author.

E-mail address: m.elkorso@u-paris10.fr (M.N. El Korso). polarized far-field sources and in [27], the authors studied the angular resolution limit for far-field sources in the presence of modeling errors. Moreover, in [28], the authors provide a novel methodology to derive an approximation of the resolution limit.

Nevertheless, to the best of our knowledge, no works have been done to quantify the resolvability of two closely spaced near-field sources in the non-uniform linear array (NULA) context for the general case $^{1}$ (general case, means that the time varying source signals, the noise variance, the range and the bearing are all unknown parameters). Consequently, the goal of this paper is to fill this lack by addressing the following question: "What is, in a nearfield source scenario, the minimum source separation required, under which two near-field sources can still be correctly resolved?"

To this end, one common tool is the distance resolution limit (DRL) of two closely spaced sources, defined as the minimum distance with respect to the sources for which allows a correct resolvability. The DRL can be derived based on one of the following three fundamental approaches: i) the analysis of the mean null spectrum [30], ii) the detection theory $[19,23$ ] (using a binary hypothesis test and a proper generalized likelihood ratio test [27]), or iii) the estimation accuracy (namely, based on the Cramér-Rao

\footnotetext{
1 In [29], the authors studied the resolvability in the near field context for the special optimistic case in which the source signals and the noise variance are assumed known, and the only unknown parameters are the range and the bearing. Whereas, in [28], the authors derived the resolution limit with respect to the bearing parameter only, without considering the range as a parameter of interest affecting the resolution.
} 
bound (CRB) tool). More precisely, in [31], Smith proposed the following DRL criterion: "Two sources are resolvable if the separation between them, denoted by $d_{N F}$, is less than the standard deviation of the separation estimation". Since the standard deviation can be approximated using the CRB by $\sqrt{\mathrm{CRB}\left(d_{N F}\right)}$ [31], thus, Smith's criterion states that the DRL is given as the particular value of the distance $d_{N F}$ between two sources which is equal to $\sqrt{\mathrm{CRB}\left(d_{N F}\right)}$. This means that the DRL is the solution of the following equation $\mathrm{DRL}=\sqrt{\mathrm{CRB}(\mathrm{DRL})}$.

It is worth noting that this DRL, which is based on the CRB, is widely and commonly used for the following reasons: i) it takes the coupling between the parameters into account, ii) it avoids the well-known drawback of the mean null spectrum approach which is valid for a specific given high-resolution algorithm and hence lacks generality [24], and finally, iii) it is related to the detection theory based approach thanks to a translator factor which is given in [11].

In order to derive the DRL, we first investigate and develop explicit closed-form expressions of the deterministic CRB for two closely spaced near-field spaced sources (which, to the best of our knowledge, has not been derived ${ }^{2}$ ). Then, we resort Smith's criterion to derive the DRL. Thus, we deduce and analyze the relationship between the minimum distance of resolvability and the minimum signal-to-noise ratio (SNR) required to resolve two closely spaced near-field sources. Finally, we propose a fast, nearly optimal, array design procedure to enhance the capacity of resolvability for a given constraint (number of sensors, array aperture, etc.). Numerical simulations are given to assess the validity of the proposed expressions (for both the deterministic CRB and the DRL) and the enhancement due to the proposed array geometry design.

For the rest part of this paper, the following notation will be used. A lowercase bold letter denotes a vector, and an uppercase one a matrix. Vectors are by default in column orientation unless specified. Upper scripts ${ }^{T}$ and ${ }^{H}$ denote, respectively, the transpose and the trans-conjugate of a matrix. The operators $\operatorname{diag}(\cdot)$, $\operatorname{adiag}(\cdot),\|\cdot\|$ and $\Re\{\cdot\}$ denote, respectively, the diagonal operator, the anti-diagonal operator, the Euclidean norm and the real part. $\operatorname{tr}\{\cdot\}$ and $\operatorname{det}\{\cdot\}$ denote the trace and the determinant of a matrix; whereas, $\odot$ and $\otimes$ denote the Hadamard and the Kronecker products, respectively. $\mathbf{I}_{L}$ denotes the identity matrix of size $L \times L .[\cdot]_{i}$ and $[\cdot]_{i, j}$ represent, respectively, the $i$-th vector element and the $(i, j)$-th matrix element. Finally, $\hat{\boldsymbol{\varepsilon}}$ denotes any asymptotically unbiased estimator of $\boldsymbol{\varepsilon}$.

This paper is structured as follows. Section 2 introduces the data model and the underlying assumptions. In Section 3, we derive the CRB of a NULA for orthogonal and non-orthogonal sources. We deduced the DRL in Section 4. Discussion of the results and conclusion are presented in Sections 5 and 6, respectively.

\section{System models}

Assume a linear (possibly non-uniform) array composed of $N$ sensors with locations $d_{0} \cdot d, d_{1} \cdot d, \cdots, d_{N-1} \cdot d$ that receives a signal emitted by two near-field, narrow-band sources $s_{1}(t)$ and $s_{2}(t)$, as shown in Fig. 1. Let $d_{n} \cdot d$ denote the location of the $(n+1)$-th sensor, in which $d$ is the unit and the reference sensor is the first one (i.e., $d_{0}=0$ ). Furthermore, for sake of simplicity, the sensor locations are expressed as multiplication of a common unit base-line $d$. This means that $d_{0}, d_{1}, \cdots, d_{N-1}$ are integers. The observed signal, $x_{n}(t)$, at the $t$-th snapshot of the $(n+1)$-th sensor is given by

$$
\begin{gathered}
x_{n}(t)=s_{1}(t) e^{j \tau_{n 1}}+s_{2}(t) e^{j \tau_{n 2}}+v_{n}(t), \\
n=0,1, \cdots, N-1, \quad t=1,2, \cdots, T
\end{gathered}
$$

\footnotetext{
2 More precisely, in [18-22], only the one single source case was under consideration.
}

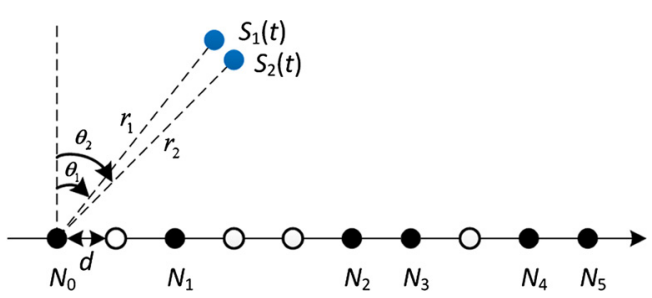

Fig. 1. Localization of two sources in the near-field region using a non-uniform linear array. Symbols $\bullet$ and o represent the position of a sensor and the position of a missing sensor, respectively.

where $v_{n}(t)$ denotes a complex circular white Gaussian noise with zero-mean and unknown variance $\sigma^{2}$, which is assumed to be uncorrelated both temporally and spatially. The $i$-th source signal, with a carrier frequency equal to $f_{0}$ is given by $s_{i}(t)=$ $\alpha_{i}(t) e^{j\left(2 \pi f_{0} t+\psi_{i}(t)\right)}, i=1,2$, where $\alpha_{i}(t)$ and $\psi_{i}(t)$ are the real amplitude and the shift phase of the $i$-th source, respectively, and $T$ is the number of snapshots. Moreover, the time delay $\tau_{n i}$ associated with the signal propagation time from the $i$-th source to the $(n+1)$-th sensor is represented as [23]

$\tau_{n i}=\frac{2 \pi r_{i}}{\lambda}\left(\sqrt{1+\frac{d_{n}^{2} d^{2}}{r_{i}^{2}}-\frac{2 d_{n} d \sin \theta_{i}}{r_{i}}}-1\right), n=0,1, \cdots, N-1$

where $\lambda$ is the signal wavelength, $r_{i}$ and $\theta_{i} \in[0, \pi / 2]$ are the range and the bearing of the $i$-th source, respectively. It is well known that if the range is inside the so-called Fresnel region [17], i.e., if

$0.62\left(\frac{d_{N-1}^{3} d^{3}}{\lambda}\right)^{1 / 2}<r_{i}<\frac{2 d_{N-1}^{2} d^{2}}{\lambda}$

then, the time delay $\tau_{n i}$ can be approximated by

$\tau_{n i} \approx \omega_{i} d_{n}+\phi_{i} d_{n}^{2}+O\left(\frac{d^{2}}{r_{i}^{2}}\right)$

in which $\omega_{i}$ and $\phi_{i}$ are the so-called electric angles for the $i$-th source which are connected to the physical parameters by the following relationships

$\omega_{i}=-2 \pi \frac{d}{\lambda} \sin \left(\theta_{i}\right)$

and

$\phi_{i}=\pi \frac{d^{2}}{\lambda r_{i}} \cos ^{2}\left(\theta_{i}\right)$

Then, based on approximation of time delay in (4), the observation at the $t$-th snapshot of the $(n+1)$-th sensor becomes

$x_{n}(t)=s_{1}(t) e^{j\left(\omega_{1} d_{n}+\phi_{1} d_{n}^{2}\right)}+s_{2}(t) e^{j\left(\omega_{2} d_{n}+\phi_{2} d_{n}^{2}\right)}+v_{n}(t)$

Consequently, the $t$-th observation vector can be expressed as

$\boldsymbol{x}(t)=\left[x_{0}(t) \cdots x_{N-1}(t)\right]^{T}=\boldsymbol{A}\left(\boldsymbol{p}_{1}, \boldsymbol{p}_{2}\right) \boldsymbol{s}(t)+\boldsymbol{v}(t)$

where $\boldsymbol{s}(t)=\left[s_{1}(t), s_{2}(t)\right]^{T}, \boldsymbol{v}(t)=\left[v_{0}(t), \cdots, v_{N-1}(t)\right]^{T}, \quad \boldsymbol{p}_{i}=$ $\left[\omega_{i}, \phi_{i}\right]^{T}$ for $i=1,2$ and $\boldsymbol{A}\left(\boldsymbol{p}_{1}, \boldsymbol{p}_{2}\right)=\left[\boldsymbol{a}\left(\omega_{1}, \phi_{1}\right), \boldsymbol{a}\left(\omega_{2}, \phi_{2}\right)\right]$. The $(n+1)$-th element of the steering vector $\boldsymbol{a}\left(\omega_{i}, \phi_{i}\right)$ is given by

$\left[\boldsymbol{a}\left(\omega_{i}, \phi_{i}\right)\right]_{n+1}=\mathrm{e}^{j\left(\omega_{i} d_{n}+\phi_{i} d_{n}^{2}\right)}$.

We assume that the unknown parameter vector is

$\boldsymbol{\varepsilon}=\left[\boldsymbol{p}_{1}^{T}, \boldsymbol{p}_{2}^{T}, \boldsymbol{\psi}_{1}^{T}, \boldsymbol{\psi}_{2}^{T}, \boldsymbol{\alpha}_{1}^{T}, \boldsymbol{\alpha}_{2}^{T}, \sigma^{2}\right]^{T}$ 
where $\boldsymbol{\psi}_{i}=\left[\psi_{i}(1), \cdots, \psi_{i}(T)\right]^{T}, \boldsymbol{\alpha}_{i}=\left[\alpha_{i}(1), \cdots, \alpha_{i}(T)\right]^{T}$. It is worth noting that the unknown vector parameter $\varepsilon$ is assumed to be deterministic and its size depends on the number of snapshots $T$.

Under the deterministic model, the joint probability distribution function (pdf) of the full observations $\chi=\left[\boldsymbol{x}^{T}(1) \cdots \boldsymbol{x}^{T}(T)\right]^{T}$, under i.i.d. assumption, is written as follows

$p(\chi \mid \boldsymbol{\varepsilon})=\frac{1}{\pi^{N T} \operatorname{det}(\boldsymbol{R})} e^{-(\chi-\mu)^{H} \boldsymbol{R}^{-1}(\chi-\mu)}$

in which $\boldsymbol{R}=\sigma^{2} \boldsymbol{I}_{N T}$ and

$\boldsymbol{\mu}=\left[\boldsymbol{s}^{T}(1) \boldsymbol{A}^{T}\left(\boldsymbol{p}_{1}, \boldsymbol{p}_{2}\right), \cdots, \boldsymbol{s}^{T}(T) \boldsymbol{A}^{T}\left(\boldsymbol{p}_{1}, \boldsymbol{p}_{2}\right)\right]^{T}$.

\section{Cramér-Rao bound definition and derivation}

Let $E\left\{(\hat{\boldsymbol{\varepsilon}}-\boldsymbol{\varepsilon})(\hat{\boldsymbol{\varepsilon}}-\boldsymbol{\varepsilon})^{T}\right\}$ be the covariance matrix of an estimate of $\boldsymbol{\varepsilon}$. Let us assume that $\hat{\boldsymbol{\varepsilon}}$ is an asymptotically unbiased estimate of the true parameter vector $\boldsymbol{\varepsilon}$, and let us, in the following, define the CRB for the considered model [32]. The covariance inequality principle states that under asymptotic conditions [33], we have

$\operatorname{MSE}\left([\hat{\boldsymbol{\varepsilon}}]_{i}\right)=E\left\{\left([\hat{\boldsymbol{\varepsilon}}]_{i}-[\boldsymbol{\varepsilon}]_{i}\right)^{2}\right\} \geq[\mathbf{C R B}(\boldsymbol{\varepsilon})]_{i, i}$

where the CRB is given as the inverse of the so-called Fisher information matrix (FIM) as follows

$\operatorname{CRB}(\varepsilon)=\operatorname{FIM}^{-1}(\varepsilon)$

Since we are working with a complex circular Gaussian observation model, the $i$-th row, $k$-th column element of the FIM for the unknown real parameter vector, given in (10), can be written as [34]:

$$
\begin{aligned}
& {\left[_{\left.\mathbf{C R B}^{-1}(\boldsymbol{\varepsilon})\right]_{i, k}}\right.} \\
& \quad=[\operatorname{FIM}(\boldsymbol{\varepsilon})]_{i, k} \\
& \quad=\operatorname{tr}\left\{\boldsymbol{R}^{-1} \frac{\partial \boldsymbol{R}}{\partial[\boldsymbol{\varepsilon}]_{i}} \boldsymbol{R}^{-1} \frac{\partial \boldsymbol{R}}{\partial[\boldsymbol{\varepsilon}]_{k}}\right\}+2 \Re\left\{\frac{\partial \boldsymbol{\mu}^{H}}{\partial[\boldsymbol{\varepsilon}]_{i}} \boldsymbol{R}^{-1} \frac{\partial \boldsymbol{\mu}}{\partial[\boldsymbol{\varepsilon}]_{k}}\right\} \\
& \quad=\frac{N T}{\sigma^{4}} \frac{\partial \sigma^{2}}{\partial[\boldsymbol{\varepsilon}]_{i}} \frac{\partial \sigma^{2}}{\partial[\boldsymbol{\varepsilon}]_{k}}+\frac{2}{\sigma^{2}} \Re\left\{\frac{\partial \boldsymbol{\mu}^{H}}{\partial[\boldsymbol{\varepsilon}]_{i}} \frac{\partial \boldsymbol{\mu}}{\partial[\boldsymbol{\varepsilon}]_{k}}\right\}
\end{aligned}
$$

\subsection{FIM derivation}

From (15) we can easily see that the noise variance $\sigma^{2}$ is decoupled from the others parameters. Thus, for simplicity and without loss of generality, we compute the FIM without taking into account the noise variance. Consequently, the variance parameter is omitted from the vector parameter $\boldsymbol{\varepsilon}$. So that the structure of the FIM is as follows (for proof, please refer to Appendix A):

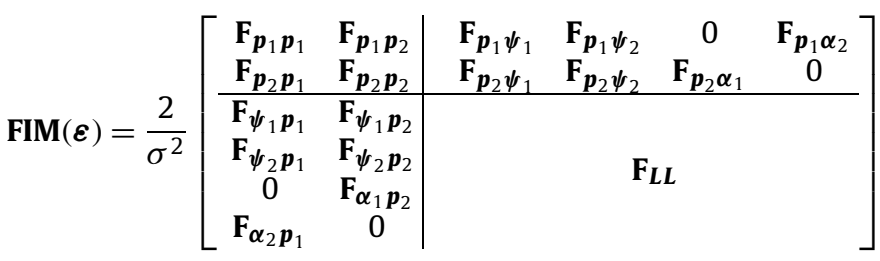

where $^{3} \boldsymbol{L}=\left[\boldsymbol{\psi}_{1}^{T}, \boldsymbol{\psi}_{2}^{T}, \boldsymbol{\alpha}_{1}^{T}, \boldsymbol{\alpha}_{2}^{T}\right]^{T}$.

\footnotetext{
3 For sake of simplicity and to avoid any misunderstanding, we used the following notation, $\mathbf{F}_{u v}$, where the lower script $\boldsymbol{u} \boldsymbol{v}$ denotes the considered part of the FIM which corresponds to the derivation according to the vector parameters $\boldsymbol{u}$ and $\boldsymbol{v}$ as shown in (7), respectively.
}

\subsection{Analytical inversion}

First, we note that the $4 T \times 4 T$ matrix $\mathbf{F}_{\boldsymbol{L} \boldsymbol{L}}$ in (16) can be partitioned into diagonal sub-blocks as

$\mathbf{F}_{\boldsymbol{L L}}=\left[\begin{array}{cc|cc}\mathbf{F}_{\psi_{1} \psi_{1}} & \mathbf{F}_{\psi_{1} \psi_{2}} & 0 & \mathbf{F}_{\boldsymbol{\psi}_{1} \boldsymbol{\alpha}_{2}} \\ \mathbf{F}_{\boldsymbol{\psi}_{2} \boldsymbol{\psi}_{1}} & \mathbf{F}_{\boldsymbol{\psi}_{2} \boldsymbol{\psi}_{2}} & \mathbf{F}_{\boldsymbol{\psi}_{2} \boldsymbol{\alpha}_{1}} & 0 \\ \hline 0 & \mathbf{F}_{\boldsymbol{\alpha}_{1} \boldsymbol{\psi}_{2}} & \mathbf{F}_{\boldsymbol{\alpha}_{1} \boldsymbol{\alpha}_{1}} & \mathbf{F}_{\boldsymbol{\alpha}_{1} \boldsymbol{\alpha}_{2}} \\ \hdashline \mathbf{F}_{\boldsymbol{\alpha}_{2} \boldsymbol{\psi}_{1}} & 0 & \overline{\mathbf{F}}_{\boldsymbol{\alpha}_{2} \boldsymbol{\alpha}_{1}} & \mathbf{F}_{\boldsymbol{\alpha}_{2} \boldsymbol{\alpha}_{2}}^{-}\end{array}\right]$

in which, the expression of each sub-block is given in Appendix A.

Second, the $\mathbf{F}_{\boldsymbol{L} \boldsymbol{L}}$ matrix is fully inverted by applying the Schur complement [35] formula twice as follows:

1) Using the Schur complement with respect to the lower right block matrix in (17) represented by the dotted line partition, one obtains

$$
\begin{aligned}
& {\left[\begin{array}{ll}
\mathbf{F}_{\boldsymbol{\alpha}_{1} \boldsymbol{\alpha}_{1}} & \mathbf{F}_{\boldsymbol{\alpha}_{1} \boldsymbol{\alpha}_{2}} \\
\mathbf{F}_{\boldsymbol{\alpha}_{2} \boldsymbol{\alpha}_{1}} & \mathbf{F}_{\boldsymbol{\alpha}_{2} \boldsymbol{\alpha}_{2}}
\end{array}\right]^{-1}=} \\
& {\left[\begin{array}{cc}
\mathbf{S}^{-1} & -\mathbf{S}^{-1} \mathbf{F}_{\boldsymbol{\alpha}_{1} \boldsymbol{\alpha}_{2}} \mathbf{F}_{\boldsymbol{\alpha}_{1} \boldsymbol{\alpha}_{1}}^{-1} \\
-\mathbf{F}_{\boldsymbol{\alpha}_{1} \boldsymbol{\alpha}_{1}}^{-1} \mathbf{F}_{\boldsymbol{\alpha}_{2} \boldsymbol{\alpha}_{1}} \mathbf{S}^{-1} & \mathbf{F}_{\boldsymbol{\alpha}_{1} \boldsymbol{\alpha}_{1}}^{-1}+\mathbf{F}_{\boldsymbol{\alpha}_{1} \boldsymbol{\alpha}_{1}}^{-1} \mathbf{F}_{\boldsymbol{\alpha}_{2} \boldsymbol{\alpha}_{1}} \mathbf{S}^{-1} \mathbf{F}_{\boldsymbol{\alpha}_{1} \boldsymbol{\alpha}_{2}} \mathbf{F}_{\boldsymbol{\alpha}_{1} \boldsymbol{\alpha}_{1}}^{-1}
\end{array}\right]}
\end{aligned}
$$

in which

$$
\mathbf{S}=\mathbf{F}_{\boldsymbol{\alpha}_{2} \boldsymbol{\alpha}_{2}}-\mathbf{F}_{\boldsymbol{\alpha}_{1} \boldsymbol{\alpha}_{2}} \mathbf{F}_{\boldsymbol{\alpha}_{1} \boldsymbol{\alpha}_{1}}^{-1} \mathbf{F}_{\boldsymbol{\alpha}_{2} \boldsymbol{\alpha}_{1}}
$$

2) Now, we use the Schur complement with respect to the lower right block matrix in (17) represented by the solid line partition and we obtain

$$
\begin{aligned}
\mathbf{F}_{\boldsymbol{L L}}^{-1} & =\left[\begin{array}{cccc}
\mathbf{F}_{\psi_{1} \psi_{1}} & \mathbf{F}_{\psi_{1} \psi_{2}} & 0 & \mathbf{F}_{\psi_{1} \boldsymbol{\alpha}_{2}} \\
\mathbf{F}_{\psi_{2} \psi_{1}} & \mathbf{F}_{\psi_{2} \psi_{2}} & \mathbf{F}_{\psi_{2} \boldsymbol{\alpha}_{1}} & 0 \\
0 & \mathbf{F}_{\boldsymbol{\alpha}_{1} \psi_{2}} & \mathbf{F}_{\boldsymbol{\alpha}_{1} \boldsymbol{\alpha}_{1}} & \mathbf{F}_{\boldsymbol{\alpha}_{1} \boldsymbol{\alpha}_{2}} \\
\mathbf{F}_{\boldsymbol{\alpha}_{2} \boldsymbol{\psi}_{1}} & 0 & \mathbf{F}_{\boldsymbol{\alpha}_{2} \boldsymbol{\alpha}_{1}} & \mathbf{F}_{\boldsymbol{\alpha}_{2} \boldsymbol{\alpha}_{2}}
\end{array}\right]^{-1} \\
& =\left[\begin{array}{cc}
\left(\mathbf{A}-\mathbf{B D}^{-1} \mathbf{C}\right)^{-1} & -\left(\mathbf{A}-\mathbf{B D}^{-1} \mathbf{C}\right)^{-1} \mathbf{B D}^{-1} \\
-\mathbf{D}^{-1} \mathbf{C}\left(\mathbf{A}-\mathbf{B D}^{-1} \mathbf{C}\right)^{-1} & \mathbf{D}^{-1}+\mathbf{D}^{-1} \mathbf{C}\left(\mathbf{A}-\mathbf{B D}^{-1} \mathbf{C}\right)^{-1} \mathbf{B D}^{-1}
\end{array}\right]
\end{aligned}
$$

where

$$
\begin{aligned}
& \mathbf{A}=\left[\begin{array}{ll}
\mathbf{F}_{\psi_{1} \psi_{1}} & \mathbf{F}_{\psi_{1} \psi_{2}} \\
\mathbf{F}_{\boldsymbol{\psi}_{2} \boldsymbol{\psi}_{1}} & \mathbf{F}_{\boldsymbol{\psi}_{2} \boldsymbol{\psi}_{2}}
\end{array}\right], \quad \mathbf{B}=\left[\begin{array}{cc}
0 & \mathbf{F}_{\boldsymbol{\psi}_{1} \boldsymbol{\alpha}_{2}} \\
\mathbf{F}_{\boldsymbol{\psi}_{2} \boldsymbol{\alpha}_{1}} & 0
\end{array}\right], \\
& \mathbf{C}=\left[\begin{array}{cc}
0 & \mathbf{F}_{\boldsymbol{\alpha}_{1} \boldsymbol{\psi}_{2}} \\
\mathbf{F}_{\boldsymbol{\alpha}_{2} \boldsymbol{\psi}_{1}} & 0
\end{array}\right] \text { and } \mathbf{D}=\left[\begin{array}{ll}
\mathbf{F}_{\boldsymbol{\alpha}_{1} \boldsymbol{\alpha}_{1}} & \mathbf{F}_{\boldsymbol{\alpha}_{1} \boldsymbol{\alpha}_{2}} \\
\mathbf{F}_{\boldsymbol{\alpha}_{2} \boldsymbol{\alpha}_{1}} & \mathbf{F}_{\boldsymbol{\alpha}_{2} \boldsymbol{\alpha}_{2}}
\end{array}\right] .
\end{aligned}
$$

3) Finally, having the inverse of $\mathbf{F}_{L L}$ matrix one can apply again this method to compute analytically the Schur complement of the lower right block of the matrix $\operatorname{FIM}(\boldsymbol{\varepsilon})$ in (16).

After tedious calculation, we are able to express in closed form the Schur complement of the matrix $\mathbf{F}_{L L}$, which corresponds to CRB $_{P \text { P }}^{-1}$ :

$\mathbf{C R B}_{\boldsymbol{P} \boldsymbol{P}}^{-1}=\frac{2}{\sigma^{2}}\left[\begin{array}{cccc}\Lambda(1,1,2) & \Gamma(1) & \Theta(1) & \Xi \\ \Gamma(1) & \Lambda(1,2,4) & \Xi & \Theta(2) \\ \Theta(1) & \Xi & \Lambda(2,1,2) & \Gamma(2) \\ \Xi & \Theta(2) & \Gamma(2) & \Lambda(2,2,4)\end{array}\right]$

in which $\boldsymbol{P}=\left[\boldsymbol{p}_{1}^{T}, \boldsymbol{p}_{2}^{T}\right]^{T}$ and functions $\Lambda, \Gamma, \Theta$ and $\Xi$ are given in Appendix B.

Consequently, instead of inverting a $(4 T+4) \times(4 T+4)$ matrix to obtain $\mathbf{C R B}_{\boldsymbol{p}_{i} \boldsymbol{p}_{k}}$, we can invert just a $2 \times 2$ matrix as follows 


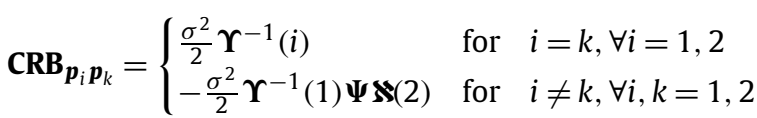

where

$\aleph(i)=\frac{1}{\Lambda(i, 1,2) \Lambda(i, 2,4)-\Gamma^{2}(i)}\left[\begin{array}{cc}\Lambda(i, 2,4) & -\Gamma(i) \\ -\Gamma(i) & \Lambda(i, 1,2)\end{array}\right]$,

$\boldsymbol{\Psi}=\left[\begin{array}{cc}\Theta(1) & \Xi \\ \Xi & \Theta(2)\end{array}\right]$ and

$\Upsilon(i)=\left[\begin{array}{cc}\Lambda(i, 1,2) & \Gamma(i) \\ \Gamma(i) & \Lambda(i, 2,4)\end{array}\right]-\boldsymbol{\Psi} \aleph(3-i) \boldsymbol{\Psi}$,

which, finally, leads to the following result

$\mathbf{C R B}_{\boldsymbol{p}_{i} \boldsymbol{p}_{i}}=\frac{\sigma^{2}}{2} \frac{1}{h(i, 1) h(i, 2)-m(i)^{2}}\left[\begin{array}{cc}h(i, 2) & -m(1) \\ -m(1) & h(i, 1)\end{array}\right]$,

$$
\forall i=1,2
$$

where

$$
\begin{aligned}
h(i, j)= & \Lambda(i, j, 2 j) \\
& +\frac{\Theta(j)(\Lambda(3-i, 3-j, 2(3-j)) \Theta(j)-\Gamma(3-i) \Xi)}{\Gamma^{2}(3-i)-\Lambda(3-i, 1,2) \Lambda(3-i, 2,4)} \\
& +\frac{\Xi(\Lambda(3-i, j, 2 j) \Xi-\Gamma(3-i) \Theta(j))}{\Gamma^{2}(3-i)-\Lambda(3-i, 1,2) \Lambda(3-i, 2,4)},
\end{aligned}
$$

and

$$
\begin{aligned}
m(i)=\Gamma(i) & +\frac{\Theta(1)(\Lambda(3-i, 2,4)) \Xi-\Gamma(3-i) \Theta(2))}{\Gamma^{2}(3-i)-\Lambda(3-i, 1,2) \Lambda(3-i, 2,4)} \\
& +\frac{\Xi(\Lambda(3-i, 1,2) \Theta(2)-\Gamma(3-i) \Xi)}{\Gamma^{2}(3-i)-\Lambda(3-i, 1,2) \Lambda(3-i, 2,4)} .
\end{aligned}
$$

\subsection{CRB for the physical parameter vector $\boldsymbol{\kappa}$}

Even if the model (7) is usually used in array signal processing, its CRB related to $\boldsymbol{\varepsilon}$ does not bring us physical information. Then, it is interesting to analyze this CRB regarding the bearing $\theta$ and range $r$.

That is, the new unknown physical parameter vector becomes

$\boldsymbol{\kappa}=\mathbf{g}(\boldsymbol{\varepsilon})=\left[\theta_{1}, r_{1}, \theta_{2}, r_{2}, \boldsymbol{\psi}_{1}^{T}, \boldsymbol{\psi}_{2}^{T}, \boldsymbol{\alpha}_{1}^{T}, \boldsymbol{\alpha}_{2}^{T}\right]^{T}$

From $\mathbf{C R B}(\boldsymbol{\varepsilon})$, we deduce $\mathbf{C R B}(\boldsymbol{\kappa})$ by using the following formula (see [36, p. 45])

$\mathbf{C R B}(\boldsymbol{\kappa})=\frac{\partial \boldsymbol{g}(\boldsymbol{\varepsilon})}{\partial \boldsymbol{\varepsilon}} \mathbf{C R B}(\boldsymbol{\varepsilon})\left[\frac{\partial \boldsymbol{g}(\boldsymbol{\varepsilon})}{\partial \boldsymbol{\varepsilon}}\right]^{T}$

where the Jacobian matrix is given by

$\frac{\partial \boldsymbol{g}(\boldsymbol{\varepsilon})}{\partial \boldsymbol{\varepsilon}}=\frac{\lambda}{\pi d}\left[\begin{array}{ccccc}\frac{1}{-2 \cos \left(\theta_{1}\right)} & 0 & 0 & 0 & 0 \\ \frac{r_{1} \sin \left(\theta_{1}\right)}{\cos ^{2}\left(\theta_{1}\right)} & \frac{-r_{1}^{2}}{d \cos ^{2}\left(\theta_{1}\right)} & 0 & 0 & 0 \\ 0 & 0 & \frac{1}{-2 \cos \left(\theta_{2}\right)} & 0 & 0 \\ 0 & 0 & \frac{r_{2} \sin \left(\theta_{2}\right)}{\cos ^{2}\left(\theta_{2}\right)} & \frac{-r_{2}^{2}}{d \cos ^{2}\left(\theta_{2}\right)} & 0 \\ 0 & 0 & 0 & 0 & \frac{\pi d}{\lambda} \boldsymbol{I}_{4 T}\end{array}\right]$

Using (27) and the Jacobian matrix above, we obtain

$\operatorname{CRB}\left(\theta_{i}\right)=\frac{\sigma^{2}}{2} \frac{\lambda^{2} h(i, 2)}{4 \pi^{2} d^{2} \cos ^{2}\left(\theta_{i}\right)\left(h(i, 1) h(i, 2)-m(i)^{2}\right)}$,

and

$\operatorname{CRB}\left(r_{i}\right)=\frac{\sigma^{2}}{2} \frac{\lambda^{2} m(i)^{2}\left(h(i, 2) d^{2} \sin ^{2}\left(\theta_{i}\right)+2 k(i) r_{i} d \sin \left(\theta_{i}\right)+h(i, 1) r_{i}^{2}\right)}{\pi^{2} d^{4} \cos ^{4}\left(\theta_{i}\right)\left(h(i, 1) h(i, 2)-m(i)^{2}\right)}$.

\subsection{The special case of two orthogonal sources}

In this subsection, we consider the particular case of two nearfield orthogonal sources. This kind of signal is commonly used in radar and radio communication to facilitate the task of separating signals [37]. From the above results, we deduce the CRB for two near-field orthogonal sources. The assumption of two orthogonal sources means that [38]

$\sum_{t=1}^{T} s_{1}^{H}(t) s_{2}(t)=0$

Substituting (31) in (18), we obtain

$\boldsymbol{\Psi}=\mathbf{0}_{2 \times 2}$

Consequently, the $\mathbf{C R B}_{\boldsymbol{p}_{i} \boldsymbol{p}_{k}}$ for near-field orthogonal sources is given by

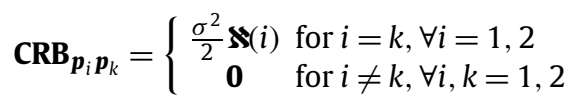

which leads to the following results

$$
\begin{aligned}
& \operatorname{CRB}\left(\omega_{i}\right)=\frac{\sigma^{2}}{2} \frac{\Lambda(i, 2,4)}{\Lambda(i, 1,2) \Lambda(i, 2,4)-\Gamma^{2}(i)} \\
& \operatorname{CRB}\left(\phi_{i}\right)=\frac{\sigma^{2}}{2} \frac{\Lambda(i, 1,2)}{\Lambda(i, 1,2) \Lambda(i, 2,4)-\Gamma^{2}(i)} \\
& \operatorname{CRB}\left(\theta_{i}\right)=\frac{\sigma^{2}}{2} \frac{\lambda^{2} \Lambda(i, 2,4)}{4 \pi^{2} d^{2} \cos ^{2}\left(\theta_{i}\right)\left[\Lambda(i, 1,2) \Lambda(i, 2,4)-\Gamma^{2}(i)\right]}
\end{aligned}
$$

and

$$
\mathrm{CRB}\left(r_{i}\right)=\frac{\sigma^{2}}{2} \frac{\lambda^{2} r_{i}^{2}\left[\Lambda(i, 2,4) d^{2} \sin ^{2}\left(\theta_{i}\right)+2 \Gamma(i) r_{i} d \sin \left(\theta_{i}\right)+\Lambda(i, 1,2) r_{i}^{2}\right]}{\pi^{2} d^{4} \cos ^{4}\left(\theta_{i}\right)\left[\Lambda(i, 1,2) \Lambda(i, 2,4)-\Gamma^{2}(i)\right]} .
$$

\section{The minimum SNR required to resolve two closely spaced near-field sources}

The aim of this section is to derive a closed-form expression of the minimum SNR, denoted by $\mathrm{SNR}_{\min }$, required to resolve two closely spaced near-field sources. By using Smith's criterion [39], the DRL is the particular value of the distance $d_{N F}$ between two sources for which $\sqrt{\mathrm{CRB}\left(d_{N F}\right)}$ is equal to $d_{N F}$, where the distance between two near field sources is defined as

$d_{N F}=\sqrt{r_{1}^{2}+r_{2}^{2}-2 r_{1} r_{2} \cos \left(\theta_{2}-\theta_{1}\right)}$.

Consequently, the new unknown physical parameter vector is

$\boldsymbol{j}(\boldsymbol{\kappa})=\left[\sqrt{r_{1}^{2}+r_{2}^{2}-2 r_{1} r_{2} \cos \left(\theta_{2}-\theta_{1}\right)}, r_{1}, \theta_{2}, r_{2}, \boldsymbol{\psi}_{1}^{T}, \boldsymbol{\psi}_{2}^{T}, \boldsymbol{\alpha}_{1}^{T}, \boldsymbol{\alpha}_{2}^{T}\right]^{T}$

As in (27), we obtain CRB $\left(d_{N F}\right)$ from the following formula

$\operatorname{CRB}\left(d_{N F}\right)=\left[\frac{\partial \boldsymbol{j}(\boldsymbol{\kappa})}{\partial \boldsymbol{\kappa}} \mathbf{C R B}(\boldsymbol{\kappa})\left[\frac{\partial \boldsymbol{j}(\boldsymbol{\kappa})}{\partial \boldsymbol{\kappa}}\right]^{T}\right]_{1,1}$

From (21), (22) and using the relation (27), one notices that $\widetilde{\mathbf{C R B}}(\boldsymbol{\kappa})=\frac{\mathbf{C R B}(\kappa)}{\sigma^{2}}$ does not depend on $\sigma^{2}$. Denoting $\widetilde{\mathrm{CRB}}\left(d_{N F}\right)=$ $\frac{\operatorname{CRB}\left(d_{N F}\right)}{\sigma^{2}}$ equation (40) can be written as

$\widetilde{\mathrm{CRB}}\left(d_{N F}\right)=\left[\frac{\partial \boldsymbol{j}(\boldsymbol{\kappa})}{\partial \boldsymbol{\kappa}} \widetilde{\mathbf{C R B}(\boldsymbol{\kappa})}\left[\frac{\partial \boldsymbol{j}(\boldsymbol{\kappa})}{\partial \boldsymbol{\kappa}}\right]^{T}\right]_{1,1}$ 


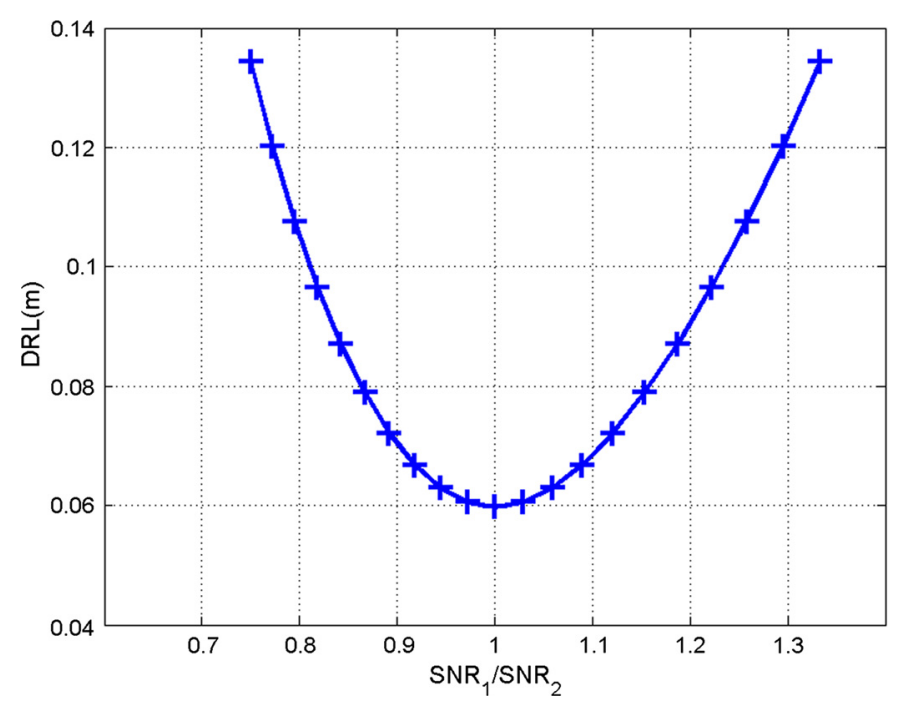

Fig. 2. DRL vs. the ratio of SNRs in the case of a ULA of 6 sensors, 100 snapshots and $\mathrm{SNR}_{1}+\mathrm{SNR}_{2}=30 \mathrm{~dB}$.

Consequently, one obtains

$$
\widetilde{\mathrm{CRB}}\left(d_{N F}\right)=\sum_{i, j=1}^{4}\left[\frac{\partial \boldsymbol{j}(\boldsymbol{\kappa})}{\partial \boldsymbol{\kappa}}\right]_{1, i}\left[\frac{\partial \boldsymbol{j}(\boldsymbol{\kappa})}{\partial \boldsymbol{\kappa}}\right]_{1, j}[\widetilde{\mathbf{C R B}}(\boldsymbol{\kappa})]_{i, j}
$$

where

$$
\left[\frac{\partial \boldsymbol{j}(\boldsymbol{\kappa})}{\partial \boldsymbol{\kappa}}\right]_{1,1}=-\left[\frac{\partial \boldsymbol{j}(\boldsymbol{\kappa})}{\partial \boldsymbol{\kappa}}\right]_{1,3}=-\frac{r_{1} r_{2} \sin \left(\theta_{2}-\theta_{1}\right)}{d_{N F}},
$$

and

$$
\left[\frac{\partial \boldsymbol{j}(\boldsymbol{\kappa})}{\partial \boldsymbol{\kappa}}\right]_{1,2}=-\left[\frac{\partial \boldsymbol{j}(\boldsymbol{\kappa})}{\partial \boldsymbol{\kappa}}\right]_{1,4}=\frac{r_{1}-r_{2} \cos \left(\theta_{2}-\theta_{1}\right)}{d_{N F}} .
$$

Let us define the SNR as

$$
\mathrm{SNR}=\frac{\left\|\boldsymbol{\alpha}_{\mathbf{1}}\right\|^{2}+\left\|\boldsymbol{\alpha}_{\mathbf{2}}\right\|^{2}}{\sigma^{2}}
$$

Furthermore, from (40) and (42), one has $\widetilde{\mathrm{CRB}}\left(d_{N F}\right)=\frac{\operatorname{CRB}\left(d_{N F}\right)}{\sigma^{2}}$. Since, $\widetilde{\mathrm{CRB}}\left(d_{N F}\right)$ is $\sigma^{2}$ independent and the $\mathrm{SNR}_{\min }$ is given by the SNR for which the following equation holds

$\sigma^{2} \widetilde{\mathrm{CRB}}(\mathrm{DRL})=r_{1}^{2}+r_{2}^{2}-2 r_{1} r_{2} \cos \left(\theta_{2}-\theta_{1}\right)$

Consequently, from (45) and (46), one obtains

$\mathrm{SNR}_{\text {min }}=\frac{\left(\left\|\boldsymbol{\alpha}_{1}\right\|^{2}+\left\|\boldsymbol{\alpha}_{2}\right\|^{2}\right) \widetilde{\mathrm{CRB}}(\mathrm{DRL})}{r_{1}^{2}+r_{2}^{2}-2 r_{1} r_{2} \cos \left(\theta_{2}-\theta_{1}\right)}$

Remark. We should precise that using the definition of the total $\mathrm{SNR}$ in the deterministic case (i.e., $\mathrm{SNR}=\mathrm{SNR}_{1}+\mathrm{SNR}_{2}$, in which $\mathrm{SNR}_{1}=\frac{\left\|\boldsymbol{\alpha}_{1}\right\|^{2}}{\sigma^{2}}$ and $\mathrm{SNR}_{2}=\frac{\left\|\boldsymbol{\alpha}_{2}\right\|^{2}}{\sigma^{2}}$ ) to characterize the $\mathrm{SNR}_{\min }$ we lose the information related to the ratio of SNRs, i.e., $\frac{S N R_{1}}{S N R_{2}}$. Consequently, one has to take a precaution using the $\mathrm{SNR}_{\min }$, since, for a given DRL one may require a higher $\mathrm{SNR}_{\min }$ if the ratio $\frac{\mathrm{SNR}_{1}}{\mathrm{SNR}_{2}}$ is far from 1 as represented in Fig. 2. Indeed, Fig. 2 shows the variation of the DRL w.r.t. $\frac{\mathrm{SNR}_{1}}{\mathrm{SNR}_{2}}$ subject to a fixed total SNR (i.e., $\mathrm{SNR}_{1}+\mathrm{SNR}_{2}=$ constant). Furthermore, we can notice, by simulation, that the smallest $S_{N R}$ min possible for a given DRL is obtained in the case of $\frac{\mathrm{SNR}_{1}}{\mathrm{SNR}_{2}}=1$.

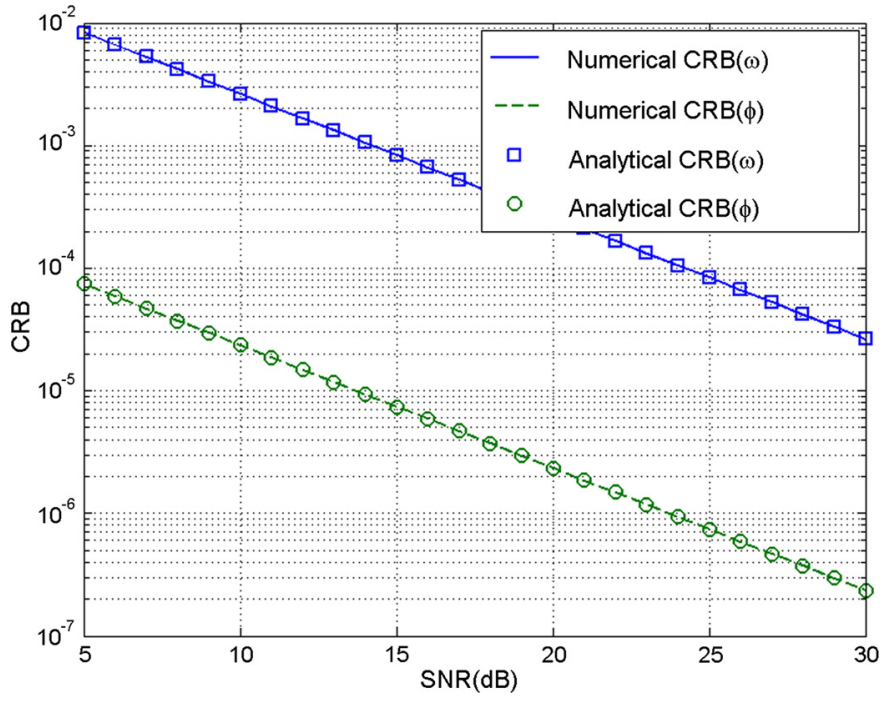

Fig. 3. Comparison between numerical and analytical $\operatorname{CRB}\left(\omega_{1}\right)$ and $\operatorname{CRB}\left(\phi_{1}\right)$ with $\left(\theta_{1}, r_{1}\right)=\left(30^{\circ}, 10 \lambda\right)$ and $\left(\theta_{2}, r_{2}\right)=\left(60^{\circ}, 20 \lambda\right)$. Lines and markers represent the numerical and analytical CRBs, respectively.

\section{Numerical investigation: CRB, DRL and geometry design}

Numerical results are presented in this section for a NULA with six sensors (with the following locations: $0,3 \cdot d, 4 \cdot d, 5 \cdot d, 6 \cdot d$, $9 \cdot d$ ) that receives a signal emitted by two sources in the Fresnel region in which their amplitudes and phases are generated from a realization of an i.i.d. Gaussian distribution with zero mean and unit variance unless specified. The number of snapshots is given by $T=100$.

\subsection{Numerical analysis of the CRB}

In this section, we analyze the proposed closed-form expressions given in Section 3 by comparing the analytical results with the numerical results to illustrate the validity of our derived CRB closed form expressions. More precisely,

- In Fig. 3, we compare the analytical CRBs given in (23) with the numerical exact CRBs given in (6). This figure shows that the proposed closed-form expressions are in a good agreement with the true numerical CRBs, which validates our derived expressions. Furthermore, we have the same results for $\operatorname{CRB}(\theta)$ and $\mathrm{CRB}(r)$ with respect to (29) and (30).

- From (24), we notice that $\forall i=1,2, h(i, 1)$ is $O\left(N^{3}\right)$, however $h(i, 2)$ is $O\left(N^{8}\right)$, which means that the estimation of the socalled second electrical angle $\phi_{i}$ is more accurate than estimating the so-called first electrical angle $\omega_{i}$. This is corroborated by Fig. 3.

- From (22) and using the inversion lemma matrix, we can quantify the effect of the estimation accuracy of one source on the other one by the following relationship

$$
\begin{aligned}
& \mathbf{C R B}_{\boldsymbol{p}_{1} \boldsymbol{p}_{1}}=\frac{\sigma^{2}}{2} \boldsymbol{\aleph}(1)+\boldsymbol{\aleph}(1) \boldsymbol{\Psi} \mathbf{C R B}_{\boldsymbol{p}_{2} \boldsymbol{p}_{2}} \boldsymbol{\Psi} \mathbf{\$}(1) \\
& =\frac{\sigma^{2}}{2} \aleph(1)\left(\mathbf{I}^{2 \times 2}+\frac{2}{\sigma^{2}} \Psi \mathbf{C R B}_{\boldsymbol{p}_{2}} \boldsymbol{p}_{2} \boldsymbol{\Psi} \aleph(1)\right)
\end{aligned}
$$

and in the same way we obtain

$$
\operatorname{CRB}_{p_{2} p_{2}}=\frac{\sigma^{2}}{2} \aleph(2)\left(I^{2 \times 2}+\frac{2}{\sigma^{2}} \boldsymbol{\Psi} \operatorname{CRB}_{p_{1}} p_{1} \Psi \aleph(2)\right)
$$

Thus, in the general case, the estimation error of the electrical angles of one source affects the electrical angles estimation of 


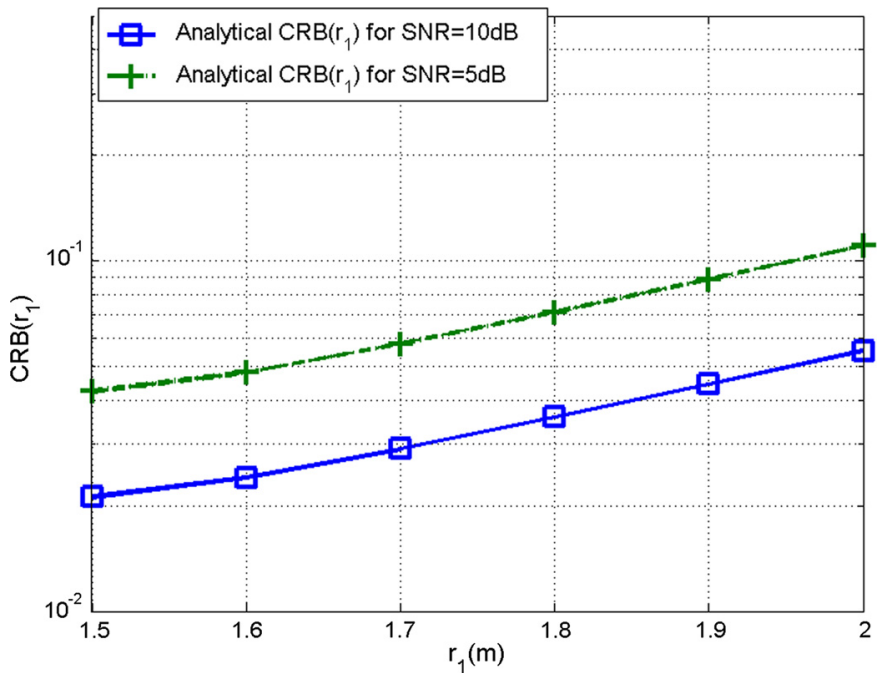

Fig. 4. Variation of the CRB w.r.t. $r_{1}$ with fixed $r_{2}=2 \mathrm{~m}$ and for equal source power.

the other source. Whereas, from (48) and (49), we note that this is not the case if the sources are orthogonal, meaning that the estimation accuracy of one source neither depends on nor affects the estimation accuracy of the other source. However, it is obvious that $\mathbf{C R B}_{\boldsymbol{p}_{i} \boldsymbol{p}_{i}}$ is affected by the position of both sources, more precisely, by the distance between the electric angles of both sources (because $\Lambda(i, k, p)$ depends on $\Delta \omega$ and $\triangle \phi$ even in the orthogonal case, and thus $\mathbf{C R B}_{\boldsymbol{p}_{i}} \boldsymbol{p}_{i}$ depends on the distance between the electrical angles).

- Unlike the case of one source, the CRB for two sources are not phase-invariant. However, we notice that for two orthogonal sources the CRB becomes phase-invariant. Numerical simulation shows the improvement due to orthogonality.

- For a sufficient number of sensors, $\mathbf{C R B}_{\boldsymbol{\theta}_{i} \boldsymbol{\theta}_{i}}$ and $\mathbf{C R B}_{\boldsymbol{r}_{i} \boldsymbol{r}_{i}}$ are $O\left(1 / N^{3}\right)$.

- $\mathbf{C R B}_{\boldsymbol{r}_{i} \boldsymbol{r}_{i}}$ is bearing-invariant and range-invariant.

- For $\lambda, r_{i} \propto d$, the dependence on the range is $O\left(r_{i}^{2}\right)$, meaning that nearer the source is, the better the estimation (keeping in mind the Fresnel constraints). This is corroborated by Fig. 4 . The dependence of the range on the bearing is $O\left(1 / \cos ^{4}\left(\theta_{i}\right)\right)$. For $\theta_{i}$ close to $\pi / 2$, we observe that $\mathbf{C R B}_{\mathbf{r}_{i}} \boldsymbol{r}_{i}$ goes to infinity but faster than $\mathbf{C R B}_{\boldsymbol{\theta}_{i} \boldsymbol{\theta}_{i}}$ (cf. Fig. 5 for $\operatorname{CRB}\left(\theta_{1}\right)$ ).

- Fig. 6 shows that, for a different value of bearing and for a fixed SNR, the higher the frequency is, the lower the $\mathbf{C R B}_{\boldsymbol{r}_{i}} \boldsymbol{r}_{i}$.

\subsection{Correlation factor}

In the following, we investigate the effect of the correlation factor on the DRL. We define the correlation factor $\xi$ between the two sources as [40]

$\xi=\frac{\boldsymbol{s}_{1}^{H} \boldsymbol{s}_{2}}{\left\|\boldsymbol{s}_{1}\right\|\left\|\boldsymbol{s}_{2}\right\|}$

in which $\boldsymbol{s}_{i}=\left[s_{i}(1), \ldots, s_{i}(T)\right]^{T}$ denotes the $i$-th signal vector. We use the same simulation parameters as in Fig. 3, expect the fact the signal sources satisfy (50) for a given $\xi$. From (34) to (37), we know that the orthogonality between two sources implies the uncoupling property. Then, since the sources are decoupled, $\xi=0$, the estimation will be improved (at least for any efficient algorithm). In Fig. 7, we compare the correlation effect of the real amplitude of $\xi$ on the DRL. Note that, for a given SNR, DRL becomes greater as the value of the real amplitude of $\xi$ increases. In other words, the smaller the correlation between both sources, the

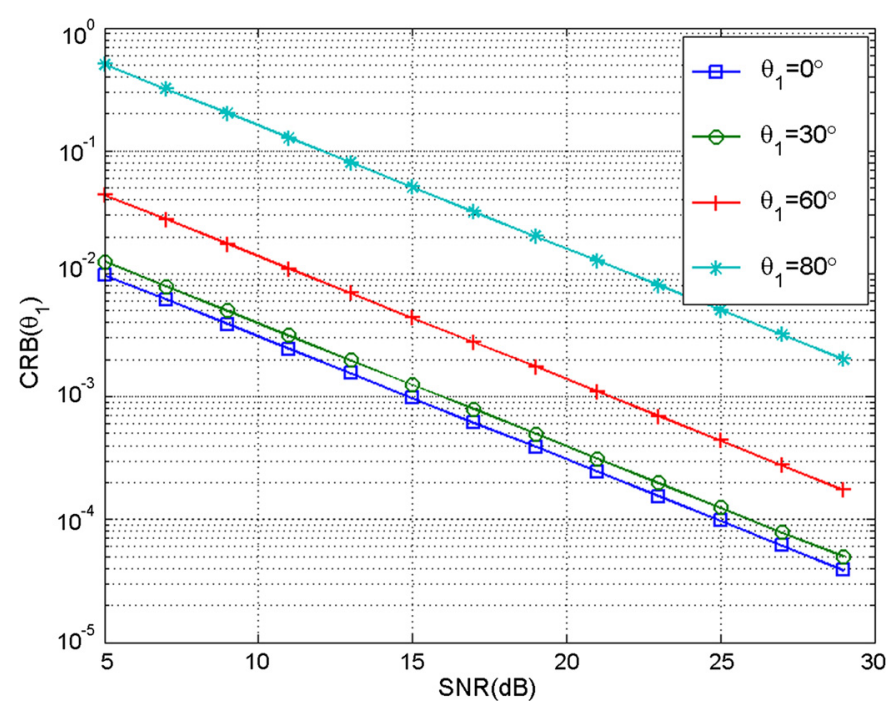

Fig. 5. $\operatorname{CRB}\left(\theta_{1}\right)$ vs. SNR for $r_{1}=10 \lambda,\left(\theta_{2}, r_{2}\right)=\left(60^{\circ}, 20 \lambda\right)$ and values of $\theta_{1}$ as shown in the legend. Lines and markers represent the analytical and numerical CRBs, respectively.

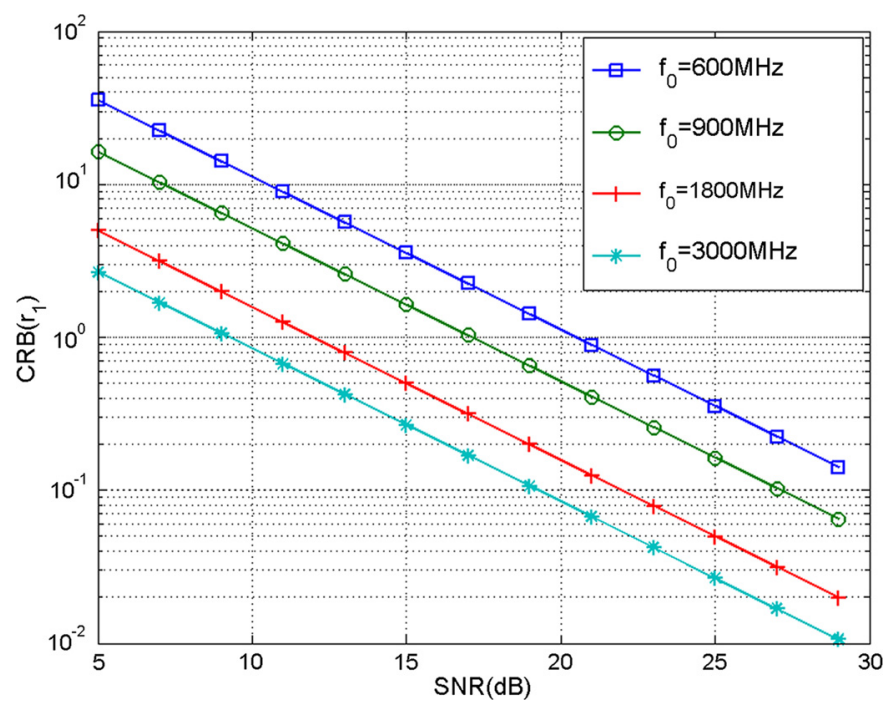

Fig. 6. $\operatorname{CRB}\left(r_{1}\right)$ vs. SNR for $\left(\theta_{1}, r_{1}\right)=\left(30^{\circ}, 10 \lambda\right),\left(\theta_{2}, r_{2}\right)=\left(60^{\circ}, 20 \lambda\right)$ and values of $f_{0}$ as shown in the legend. Lines and markers represent the analytical and numerical CRBs, respectively.

better the resolution limit performance. Meanwhile, DRL achieves its lowest value when $\xi=0$, which means that two signals enjoy a minimum (best) resolution limit between them if they have the orthogonal relationship.

\section{3. $S N R_{\min }$ behavior w.r.t. central angle}

Let us define the central angle as

$\theta_{c}=\frac{\theta_{1}+\theta_{2}}{2}$

In Fig. 8, we have plotted the $\mathrm{SNR}_{\min }$ required to resolves two closely spaced sources for different values of $\theta_{c}$. It should be noted that for a given resolution limit, as the central angle $\theta_{c}$ increases, the minimum signal to noise ratio required increases also.

\subsection{Minimum resolution limit boundary}

In practical applications, one may need the knowledge of the physical boundary, which delimits the resolvability region (mean- 


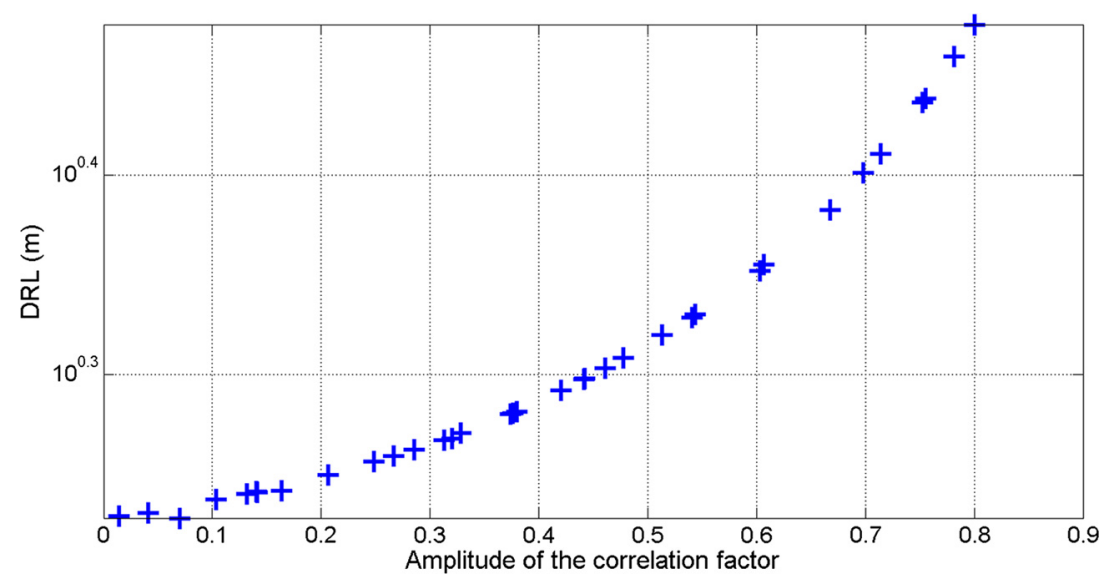

Fig. 7. The effect of the amplitude of the correlation factor on the DRL.

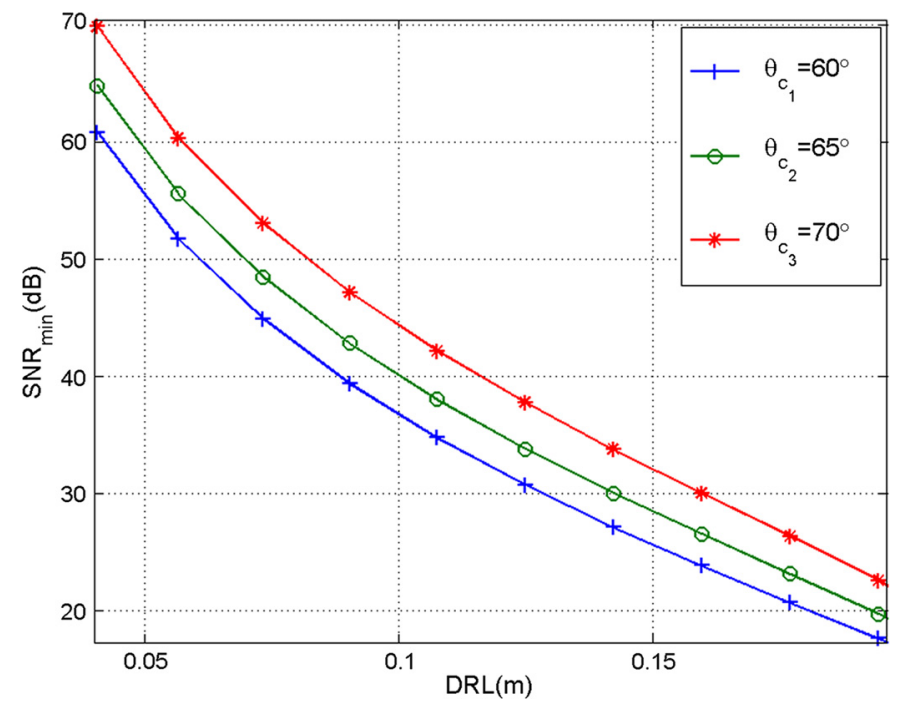

Fig. 8. The $\mathrm{SNR}_{\min }$ versus the distance curves from a non-uniform array for various central angles.

ing the region for which two near field sources are resolvable). Although this question is worth consideration, to the best of our knowledge, no work has been done on it in the literature. In this subsection, the minimum resolution limit boundary for two sources is investigated and discussed for different SNRs.

Following similar steps as those employed in Section 4, we obtain the minimum resolution limit boundary of two sources for different SNR value. Such boundary is represented in Fig. 9, in which the star symbol represents the coordinates of the first source w.r.t. the reference sensor $\left(x_{0}, y_{0}\right)=(0,0)$, in which $\left(\theta_{1}, r_{1}\right)=\left(45^{\circ}, 6 \lambda\right)$, $\left\|\boldsymbol{\alpha}_{1}\right\|^{2}=1$ and $\left\|\boldsymbol{\alpha}_{2}\right\|^{2}=1$. For different SNR, the second source must be outside this boundary to ensure a correct resolvability given by Smith's criterion. As expected, one notices that for higher SNR, one obtains a small boundary. Furthermore, we can notice that this boundary is more bearing sensitive than range sensitive. As said before, this boundary is a physical limitation. Nevertheless, this latter can be compensated by a proper array geometry design which is the aim of the next section.

\subsection{Effect of sensors' position}

The remaining part of this section is devoted to the numerical analysis of the effect of sensor array geometry on the DRL. Assume $\left(\theta_{1}, r_{1}\right)=\left(30^{\circ}, 6 \lambda\right),\left(\theta_{2}, r_{2}\right)=\left(60^{\circ}, 10 \lambda\right)$ and $\left\|\boldsymbol{\alpha}_{1}\right\|^{2}=\left\|\boldsymbol{\alpha}_{2}\right\|^{2}=1$.

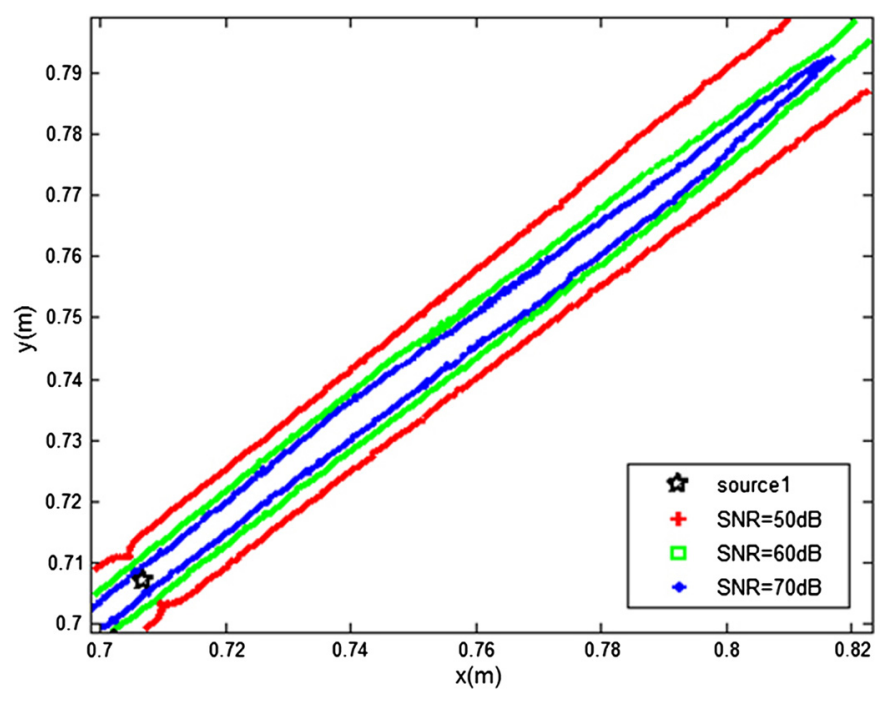

Fig. 9. DRL boundary for 6-sensors.

Table 1

Different array geometric configurations.

\begin{tabular}{lllllllllll}
\hline Array type & \multicolumn{10}{l}{ Geometric configuration } \\
\hline Type 1 & $\bullet$ & $\bullet$ & $\bullet$ & $\bullet$ & $\bullet$ & $\circ$ & $\circ$ & $\circ$ & $\circ$ & $\bullet$ \\
Type 2 & $\bullet$ & $\circ$ & $\bullet$ & $\circ$ & $\bullet$ & $\circ$ & $\bullet$ & $\circ$ & $\bullet$ & $\bullet$ \\
Type 3 & $\bullet$ & $\circ$ & $\circ$ & $\circ$ & $\circ$ & $\bullet$ & $\bullet$ & $\bullet$ & $\bullet$ & $\bullet$ \\
Type 4 & $\bullet$ & $\circ$ & $\circ$ & $\bullet$ & $\bullet$ & $\bullet$ & $\bullet$ & $\circ$ & $\circ$ & $\bullet$ \\
Type 5 & $\bullet$ & $\bullet$ & $\bullet$ & $\bullet$ & $\bullet$ & $\bullet$ & $\bullet$ & $\bullet$ & $\bullet$ & $\bullet$ \\
\hline
\end{tabular}

Keep the array aperture $A=9$ (in unit of $d=\lambda / 2$ ) fixed and $N=6$, there are 10 positions available for six sensors.

In Fig. 10, we plot the DRL which compares the impact of the different sensor array geometry w.r.t. $\mathrm{SNR}_{\min }$. Five types of array configurations are considered, as shown in Table 1. Type 1 to type 4 are listed in Table 1 with $N=6$ sensors, Type 5 is an ULA configuration with $N=10$ sensors.

From comparison of simulation results, one can further notice that

- For the same array aperture, same SNR and same number of sensors, the DRL is greatly affected by the array geometric configuration.

- By comparing the results given from Type 1 to Type 4 , the configuration that puts two sensors at the extremity and the rest in the middle seems to be the best geometry configuration (at least, it is the case for $A=9$ and $N=6$ sensors). 


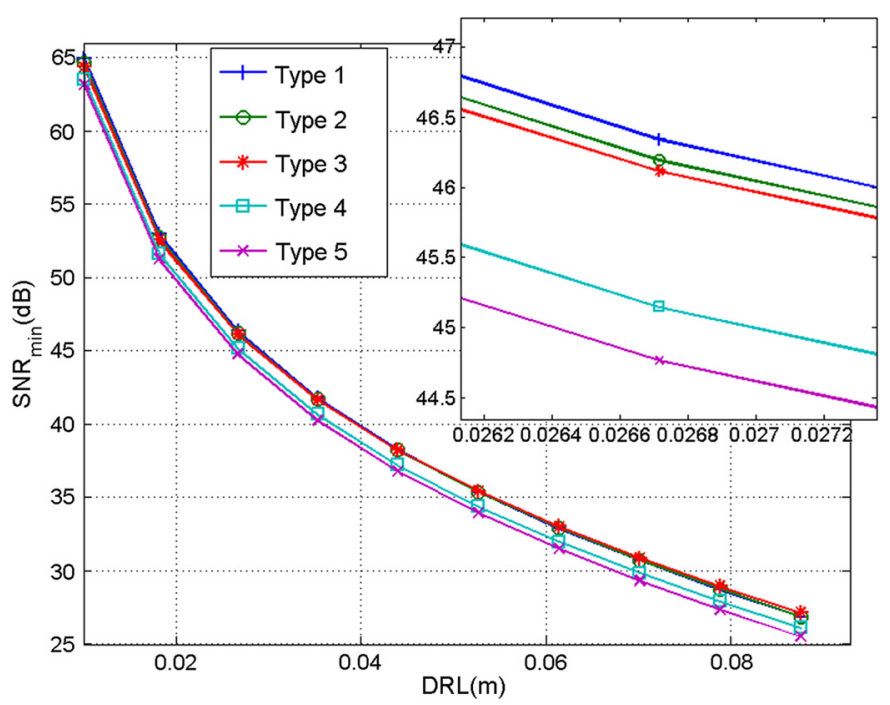

Fig. 10. The required $\mathrm{SNR}_{\min }$ to resolve two unknown closely sources for different array geometries.

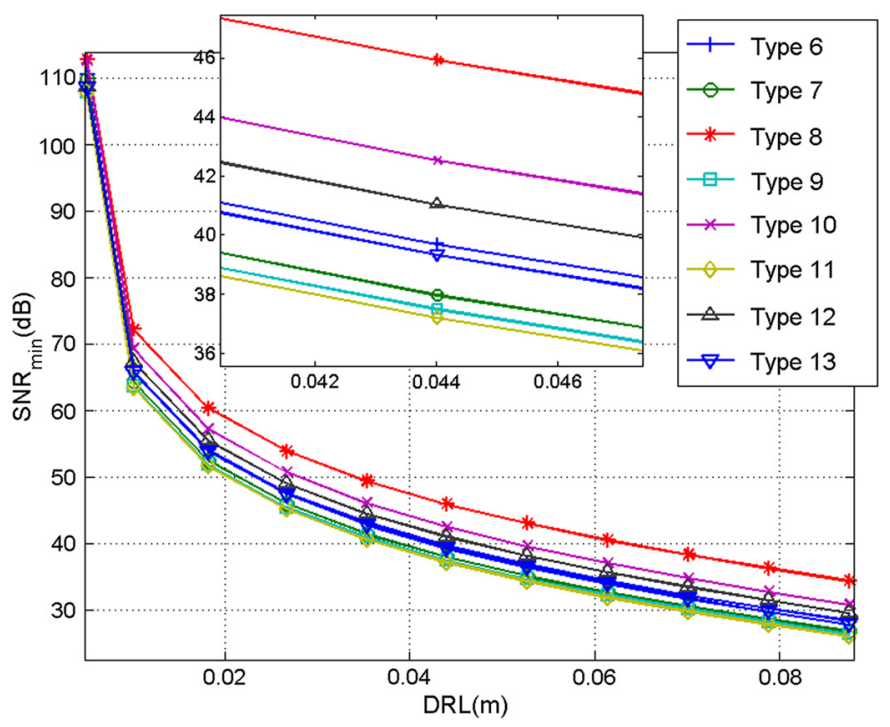

Fig. 11. The required $S N R_{\min }$ to resolve two unknown closely sources for the best $\&$ worst performance of different sensors.

\section{Table 2}

The best and worst array geometric configurations for different number of sensors.

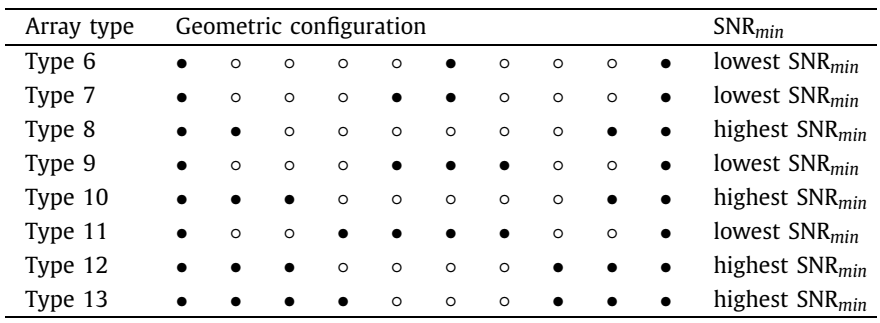

- Type 4 , with six sensors, is slightly worse than the ULA configuration with 10 sensors.

Furthermore, to better comprehend the resolvability performance as a function of array geometric configuration, consider Fig. 11, where we compare the minimum signal to noise ratio with respect to the best and worst performance of different number of sensors. The considered type of sensor geometry configuration is list in Table 2.
From this figure, we can see that the Type 11 represents the best achievable performance, and Type 8 has the worst performance. Furthermore, on one hand, we can notice that with the same array aperture, Type 6 (with 3 sensors) and Type 13 (with 7 sensors) have, approximately, the same performance (Type 6 is only $0.2 \mathrm{~dB}$ worse than the Type 13 ); and, on the other hand, one can notice that Type 6 (with 3 sensors) has a better performance in regard to Type 12 (which has 6 sensors) by $1.5 \mathrm{~dB}$. This means that depending on the array geometry, one can have better performance using a less number of sensors. The relatively poor performance of the type $8,10,12$ and 13 is due to the sensor geometry configuration. Consequently, with the same aperture, one can optimally design the array using the proposed method below, in order to obtain better performance even with less sensors.

\subsection{Sensors array geometry design}

As discussed above, the resolvability is not only constrained by the number of sensors, but also by the array geometry, which affects the optimal resolution capability.

In the following, we give a solution to design an optimal array in order to enhance the resolvability and/or to compensate the performance loss due to the reduction or failure of certain sensors.

The optimal method is given by minimizing $\mathrm{SNR}_{\min }$ over sensors' position among all possibilities using the derived expression (47). However, this scheme is not feasible for a model with large aperture and small number of sensors. More precisely, for aperture of size $A$ and $N$ sensors, one has $C_{A+1}^{N}$ possibilities. To overcome this drawback we propose a sub-optimal fast procedure as follows.

Step 1: We place two sensors at each extremity to obtain the largest aperture that leads to the best resolution.

Step 2: We place only one sensor by minimizing $\mathrm{SNR}_{\min }$ with respect to the $A-2$ remaining positions.

Step 3: We iterate the second step by placing the $(n+1)$-th sensor at once by minimizing sequentially the $\mathrm{SNR}_{\min }$ with respect to the $A-n+1$ remaining positions.

In the following part, we show that the proposed sub-optimal method produces a nearly optimal result with low complexity. More precisely, the complexity cost ratio of the optimal method over the proposed sub-optimal method is given by

$$
\begin{aligned}
f(N, A) & =\frac{N_{\text {opt }}}{N_{\text {prop }}} \\
& =\frac{2(A+1) !}{N !(A-N+1) !(A(A-1)-(A-N+1)(A-N+2))}
\end{aligned}
$$

where $N_{\text {opt }}$ means the number of required iterations by using the optimal method and $N_{\text {prop }}$ means that by using the proposed suboptimal method.

In the examples studied in this section, we consider several cases: Case 1) $N=4, A=7$; Case 2) $N=5, A=8$, so that we may compare the results of the two methods, as shown in Fig. 12. One notes that our proposed method is nearly identical to the optimal method. In addition, from (52), we have $f(4,7)=6$ and $f(5,8)=7$, which means that the proposed method is six to seven times faster than the optimal one. If we consider a more important aperture, e.g., for $N=6, A=25$, we notice that the proposed method decreases significantly the complexity cost by $f(6,25)=$ 2558 times compared with the optimal one with the same performance. Consequently, we notice that the proposed method is of low complexity, especially for the design of sparse arrays.

One should note that for the above discussion, we assume that derived closed form expression of $\mathrm{SNR}_{\min }$ is given. If no expression of the $\mathrm{SNR}_{\min }$ is given, one has, first, to numerically inverse 


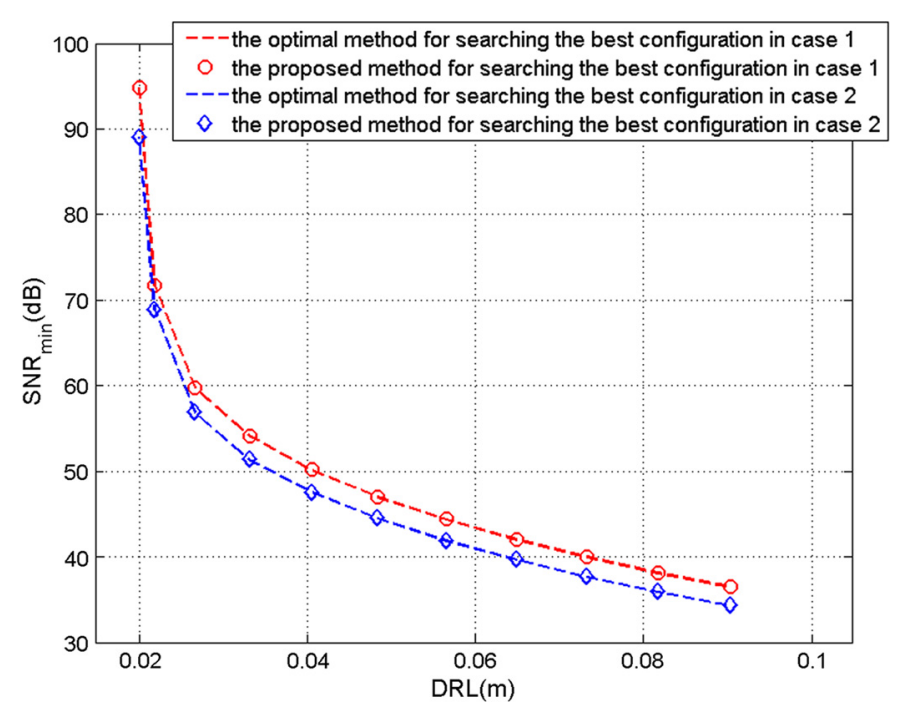

Fig. 12. Comparison between two methods of searching the optimal array geometry configuration.

the FIM (of size $4(T+1) \times 4(T+1))$ which is costly and which increases dramatically with the number of snapshots and then apply the Smith's criterion.

\section{Conclusion}

In this paper, we derived an explicit closed-form expression of the deterministic Cramér-Rao bound for two closely spaced nearfield sources for non-uniform linear array. Taking advantage of these expressions, we deduced the theoretical statistical resolution limit and the minimum signal to noise ratio required to resolve two closely spaced near-field sources. These expressions present two advantages. i) First, they avoid computationally costly numerical inversion of the Fisher information matrix, which increases dramatically with the number of snapshots. ii) Second, we provide a detailed discussion on several enlightening properties of the statistical resolution limit revealed by our expressions, with an emphasis on the effect of the given scenario (e.g., the number of sensors, the correlation factor, the minimum resolution limit boundary, the array geometry, etc.) on the resolvability. Furthermore, we present numerical analysis to corroborate the proposed theoretical expressions. Finally, based on the statistical resolution limit, we proposed a novel, fast method, which is nearly optimal, to find the best sensor arrangement leading to the best achievable resolution.

\section{Acknowledgments}

This work has been supported by the iCODE institute, research project of the IDEX Paris-Saclay (ANR-11-IDEX-0003-02), by the MAGELLAN project (ANR-14-CE23-0004-01) and by the European Network of excellence NEWCOM $\sharp$.

\section{Appendix A}

In this appendix, we present the main steps leading to (16). Consider the $(i, k)$-th element of the FIM for the unknown real parameter vector (10). We first obtain the following derivatives (for $i=1,2$ )

$$
\begin{aligned}
& \frac{\partial \boldsymbol{\mu}}{\partial \omega_{i}}=j \boldsymbol{s}_{i} \otimes \boldsymbol{d} \odot \boldsymbol{a}\left(\omega_{i}, \phi_{i}\right) \\
& \frac{\partial \boldsymbol{\mu}}{\partial \phi_{i}}=j \boldsymbol{s}_{i} \otimes \boldsymbol{d} \odot \boldsymbol{d} \odot \boldsymbol{a}\left(\omega_{i}, \phi_{i}\right)
\end{aligned}
$$

$\frac{\partial \boldsymbol{\mu}}{\partial \psi_{i}}=j \boldsymbol{s}_{i} \otimes \boldsymbol{a}\left(\omega_{i}, \phi_{i}\right)$

$\frac{\partial \boldsymbol{\mu}}{\partial \alpha_{i}}=\tilde{\boldsymbol{s}}_{i} \otimes \boldsymbol{a}\left(\omega_{i}, \phi_{i}\right)$

in which $\boldsymbol{\mu}=\left[\boldsymbol{s}^{T}(1) \boldsymbol{A}^{T}(\boldsymbol{\omega}, \boldsymbol{\phi}), \cdots, \boldsymbol{s}^{T}(T) \boldsymbol{A}^{T}(\boldsymbol{\omega}, \boldsymbol{\phi})\right]^{T},\left[\boldsymbol{s}_{i}\right]_{t}=s_{i}(t)$ and $\left[\tilde{\boldsymbol{s}}_{i}\right]_{t}=e^{j\left(2 \pi f_{0} t+\psi_{i}(t)\right)}$ for $t=1, \ldots, T$.

Let us denote

$f(m, \Delta \omega, \Delta \phi)=\sum_{n=0}^{N-1} d_{n}^{m} e^{j\left(\Delta \omega d_{n}+\Delta \phi d_{n}^{2}\right)}$

in which for sake of simplicity $f(i)$ will be used instead of $f(i, 0,0), \Delta \omega=\omega_{1}-\omega_{2}$ and $\Delta \phi=\phi_{1}-\phi_{2}$. Thus, replacing the above expressions, one has

$\frac{\partial \boldsymbol{\mu}^{\boldsymbol{H}}}{\partial \omega_{1}} \frac{\partial \boldsymbol{\mu}}{\partial \omega_{1}}=\sum_{n=0}^{N-1} d_{n}^{2} \sum_{t=1}^{T} a_{1}^{2}(t)=\left\|\boldsymbol{\alpha}_{\mathbf{1}}\right\|^{2} f(2)$,

$\frac{\partial \boldsymbol{\mu}^{\boldsymbol{H}}}{\partial \phi_{1}} \frac{\partial \boldsymbol{\mu}}{\partial \phi_{1}}=\sum_{n=0}^{N-1} d_{n}^{4} \sum_{t=1}^{T} a_{1}^{2}(t)=\left\|\boldsymbol{\alpha}_{\mathbf{1}}\right\|^{2} f(4)$,

$\frac{\partial \boldsymbol{\mu}^{\boldsymbol{H}}}{\partial \omega_{1}} \frac{\partial \boldsymbol{\mu}}{\partial \phi_{1}}=\frac{\partial \boldsymbol{\mu}^{\boldsymbol{H}}}{\partial \phi_{1}} \frac{\partial \boldsymbol{\mu}}{\partial \omega_{1}}=\sum_{n=0}^{N-1} d_{n}^{3} \sum_{t=1}^{T} a_{1}^{2}(t)=\left\|\boldsymbol{\alpha}_{\mathbf{1}}\right\|^{2} f(3)$,

and

$\boldsymbol{F}_{\boldsymbol{p}_{1} \boldsymbol{p}_{1}}=\left\|\boldsymbol{\alpha}_{\mathbf{1}}\right\| \otimes\left[\begin{array}{ll}f(2) & f(3) \\ f(3) & f(4)\end{array}\right]$.

Hence, one obtains

$$
\begin{aligned}
\frac{\partial \boldsymbol{\mu}^{\boldsymbol{H}}}{\partial \omega_{1}} \frac{\partial \boldsymbol{\mu}}{\partial \omega_{2}} & =\frac{\partial \boldsymbol{\mu}^{\boldsymbol{H}}}{\partial \omega_{2}} \frac{\partial \boldsymbol{\mu}}{\partial \omega_{1}} \\
& =\Re\left\{\sum_{n=0}^{N-1} d_{n}^{2} e^{-j\left(\Delta \omega d_{n}+\Delta \phi d_{n}^{2}\right)} \sum_{t=1}^{T} s_{1}^{H}(t) s_{2}(t)\right\} \\
& =\Re\left\{\left\langle\boldsymbol{s}_{2}, \boldsymbol{s}_{1}\right\rangle f(2, \Delta \omega, \Delta \phi)\right\} \\
\frac{\partial \boldsymbol{\mu}^{\boldsymbol{H}}}{\partial \omega_{1}} \frac{\partial \boldsymbol{\mu}}{\partial \phi_{2}} & =\frac{\partial \boldsymbol{\mu}^{\boldsymbol{H}}}{\partial \phi_{1}} \frac{\partial \boldsymbol{\mu}}{\partial \omega_{2}} \\
& =\Re\left\{\sum_{n=0}^{N-1} d_{n}^{3} e^{-j\left(\Delta \omega d_{n}+\Delta \phi d_{n}^{2}\right)} \sum_{t=1}^{T} s_{1}^{H}(t) s_{2}(t)\right\} \\
\frac{\partial \boldsymbol{\mu}^{\boldsymbol{H}}}{\partial \omega_{2}} \frac{\partial \boldsymbol{\mu}}{\partial \phi_{1}} & =\frac{\partial \boldsymbol{\mu}^{\boldsymbol{H}}}{\partial \phi_{2}} \frac{\partial \boldsymbol{\mu}}{\partial \omega_{1}} \\
& =\Re\left\{\sum_{n=0}^{N-1} d_{n}^{3} e^{j\left(\Delta \omega d_{n}+\Delta \phi d_{n}^{2}\right)} \sum_{t=1}^{T} s_{2}^{H}(t) s_{1}(t)\right\} \\
& =\Re\left\{\left\langle\boldsymbol{s}_{2}, \boldsymbol{s}_{1}\right\rangle f(3, \Delta \omega, \Delta \phi)\right\} \\
\frac{\partial \boldsymbol{\mu}^{\boldsymbol{H}}}{\partial \phi_{1}} \frac{\partial \boldsymbol{\mu}}{\partial \phi_{2}} & =\frac{\partial \boldsymbol{\mu}}{\partial \phi_{2}} \frac{\partial \boldsymbol{\mu}}{\partial \phi_{1}}\left\{\sum_{n=0}^{N-1} d_{n}^{4} e^{-j\left(\Delta \omega d_{n}+\Delta \phi d_{n}^{2}\right)} \sum_{t=1}^{T} s_{1}^{H}(t) s_{2}(t)\right\} \\
& \left.\left.=\boldsymbol{s}_{2}, \boldsymbol{s}_{1}\right\rangle f(4, \Delta \omega, \Delta \phi)\right\}
\end{aligned}
$$

After some rearrangement and calculation, one obtains the following $2 \times 2$ matrices

$$
\begin{aligned}
\boldsymbol{F}_{\boldsymbol{p}_{1} \boldsymbol{p}_{2}} & =\boldsymbol{F}_{\boldsymbol{p}_{2} \boldsymbol{p}_{1}} \\
& =\Re\left\{\left(\boldsymbol{s}_{1} \odot \boldsymbol{s}_{2}^{H}\right) \otimes\left[\begin{array}{ll}
f(2, \Delta \omega, \Delta \phi) & f(3, \Delta \omega, \Delta \phi) \\
f(3, \Delta \omega, \Delta \phi) & f(4, \Delta \omega, \Delta \phi)
\end{array}\right]\right\}
\end{aligned}
$$


$\frac{\partial \boldsymbol{\mu}^{\boldsymbol{H}}}{\partial \omega_{2}} \frac{\partial \boldsymbol{\mu}}{\partial \omega_{2}}=\sum_{n=0}^{N-1} d_{n}^{2} \sum_{t=1}^{T} \alpha_{1}^{2}(t)$,

$\frac{\partial \boldsymbol{\mu}^{\boldsymbol{H}}}{\partial \phi_{2}} \frac{\partial \boldsymbol{\mu}}{\partial \phi_{2}}=\sum_{n=0}^{N-1} d_{n}^{4} \sum_{t=1}^{T} \alpha_{1}^{2}(t)$,

$\frac{\partial \boldsymbol{\mu}^{\boldsymbol{H}}}{\partial \omega_{2}} \frac{\partial \boldsymbol{\mu}}{\partial \phi_{2}}=\frac{\partial \boldsymbol{\mu}^{\boldsymbol{H}}}{\partial \phi_{2}} \frac{\partial \boldsymbol{\mu}}{\partial \omega_{2}}=\sum_{n=0}^{N-1} d_{n}^{3} \sum_{t=1}^{T} \alpha_{1}^{2}(t)$,

and

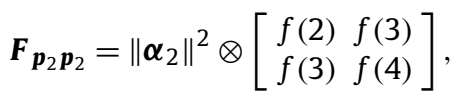

and the $2 \times T$ matrices are given by (for $i=1,2$ )

$$
\begin{aligned}
& \boldsymbol{F}_{\boldsymbol{p}_{i} \psi_{i}}=\boldsymbol{F}_{\psi_{i} \boldsymbol{p}_{i}}^{T} \\
& =\left(\boldsymbol{\alpha}_{i} \odot \boldsymbol{\alpha}_{i}\right)^{T} \otimes\left[\begin{array}{l}
f(1) \\
f(2)
\end{array}\right] \\
& =\left[\begin{array}{lll}
\alpha_{i}^{2}(1) f(1) & \cdots & \alpha_{i}^{2}(T) f(1) \\
\alpha_{i}^{2}(1) f(2) & \cdots & \alpha_{i}^{2}(T) f(2)
\end{array}\right] \\
& \boldsymbol{F}_{\boldsymbol{p}_{1} \boldsymbol{\psi}_{2}}=\boldsymbol{F}_{\boldsymbol{p}_{2} \boldsymbol{\psi}_{1}}=\boldsymbol{F}_{\psi_{2} \boldsymbol{p}_{1}}^{T}=\boldsymbol{F}_{\boldsymbol{\psi}_{1} \boldsymbol{p}_{2}}^{T} \\
& =\Re\left\{\left(\boldsymbol{s}_{1} \odot \boldsymbol{s}_{2}^{H}\right) \otimes\left[\begin{array}{l}
f(1, \Delta \omega, \Delta \phi) \\
f(2, \Delta \omega, \Delta \phi)
\end{array}\right]\right\} \\
& =\left[\begin{array}{llll}
\alpha_{1}(1) \alpha_{2}(1) \beta(0,1,1,1) & \cdots & \alpha_{1}(T) \alpha_{2}(T) \beta(0,1,1, T) \\
\alpha_{1}(1) \alpha_{2}(1) \beta(0,1,2,1) & \cdots & \alpha_{1}(T) \alpha_{2}(T) \beta(0,1,2, T)
\end{array}\right] \\
& \boldsymbol{F}_{\boldsymbol{p}_{i} \boldsymbol{\alpha}_{i}}=\boldsymbol{F}_{\boldsymbol{\alpha}_{i} \boldsymbol{p}_{i}}=\boldsymbol{F}_{\psi_{i} \boldsymbol{\alpha}_{i}}=\boldsymbol{F}_{\boldsymbol{\alpha}_{i} \psi_{i}}=0, i=1,2 \\
& \boldsymbol{F}_{\boldsymbol{p}_{1} \boldsymbol{\alpha}_{2}}=\boldsymbol{F}_{\boldsymbol{\alpha}_{2}}^{T} \boldsymbol{p}_{1} \\
& =\Re\left\{j\left(\boldsymbol{s}_{1} \odot \boldsymbol{s}_{2}^{H} \odot \overline{\boldsymbol{\alpha}}_{2}\right) \otimes\left[\begin{array}{l}
f(1, \Delta \omega, \Delta \phi) \\
f(2, \Delta \omega, \Delta \phi)
\end{array}\right]\right\} \\
& =\left[\begin{array}{lll}
\alpha_{1}(1) \beta(1,1,1,1) & \cdots & \alpha_{1}(T) \beta(1,1,1, T) \\
\alpha_{1}(1) \beta(1,1,2,1) & \cdots & \alpha_{1}(T) \beta(1,1,2, T)
\end{array}\right] \\
& \boldsymbol{F}_{\boldsymbol{p}_{2} \boldsymbol{\alpha}_{1}}=\boldsymbol{F}_{\boldsymbol{\alpha}_{1} \boldsymbol{p}_{2}}^{T} \\
& =\Re\left\{-j\left(\boldsymbol{s}_{1} \odot \boldsymbol{s}_{2}^{H} \odot \overline{\boldsymbol{\alpha}}_{1}\right) \otimes\left[\begin{array}{l}
f(1, \Delta \omega, \Delta \phi) \\
f(2, \Delta \omega, \Delta \phi)
\end{array}\right]\right\} \\
& =\left[\begin{array}{lll}
-\alpha_{2}(1) \beta(1,1,1,1) & \cdots & -\alpha_{2}(T) \beta(1,1,1, T) \\
-\alpha_{2}(1) \beta(1,1,2,1) & \cdots & -\alpha_{2}(T) \beta(1,1,2, T)
\end{array}\right]
\end{aligned}
$$

and the $T \times T$ diagonal matrices (for $i=1,2$ )

$$
\begin{aligned}
\boldsymbol{F}_{\boldsymbol{\alpha}_{i} \boldsymbol{\alpha}_{i}} & =f(0) \boldsymbol{I}_{T} \\
\boldsymbol{F}_{\boldsymbol{\alpha}_{1} \boldsymbol{\alpha}_{2}} & =\boldsymbol{F}_{\boldsymbol{\alpha}_{2} \boldsymbol{\alpha}_{1}} \\
& =\Re\left\{\operatorname{diag}\left(\boldsymbol{s}_{1} \odot \boldsymbol{s}_{2}^{H} \odot \overline{\boldsymbol{\alpha}}_{2} \odot \overline{\boldsymbol{\alpha}}_{1}\right) f(0, \Delta \omega, \Delta \phi)\right\} \\
\boldsymbol{F}_{\psi_{i} \psi_{i}} & =\operatorname{diag}\left(\boldsymbol{\alpha}_{1} \odot \boldsymbol{\alpha}_{2}\right) f(0), i=1,2 \\
\boldsymbol{F}_{\psi_{1} \psi_{2}} & =\boldsymbol{F}_{\psi_{2} \boldsymbol{\psi}_{1}}=\Re\left\{\operatorname{diag}\left(\boldsymbol{s}_{1} \odot \boldsymbol{s}_{2}^{H}\right) f(0, \Delta \omega, \Delta \phi)\right\} \\
\boldsymbol{F}_{\psi_{1} \boldsymbol{\alpha}_{2}} & =\boldsymbol{F}_{\boldsymbol{\alpha}_{2} \psi_{1}}=\Re\left\{j \operatorname{diag}\left(\boldsymbol{s}_{1} \odot \boldsymbol{s}_{2}^{H} \odot \overline{\boldsymbol{\alpha}}_{2}\right) f(0, \Delta \omega, \Delta \phi)\right\} \\
\boldsymbol{F}_{\psi_{2} \boldsymbol{\alpha}_{1}} & =\boldsymbol{F}_{\boldsymbol{\alpha}_{1} \psi_{2}}=\Re\left\{-j \operatorname{diag}\left(\boldsymbol{s}_{1} \odot \boldsymbol{s}_{2}^{H} \odot \overline{\boldsymbol{\alpha}}_{1}\right) f(0, \Delta \omega, \Delta \phi)\right\}
\end{aligned}
$$

\section{Appendix B}

In this appendix we give the expression of functions $\Lambda, \Gamma, \Theta$ and $\Xi$ :

$$
\begin{aligned}
& \Lambda(i, k, p)=\left\|\alpha_{i}\right\|^{2} f(p) \\
& \quad-\sum_{t=1}^{T} \frac{a_{i}^{2}(t)\left(f(0) f^{2}(k)-2 f(k) v(1, k+1, t)+f(0)\|f(k, \Delta \omega, \Delta \phi)\|^{2}\right)}{\gamma},
\end{aligned}
$$$$
\Gamma(i)=\left\|\alpha_{i}\right\|^{2} f(3)
$$$$
-\sum_{t=1}^{T} \frac{a_{i}^{2}(t)(f(0) f(1) f(2)+f(0) v(2,3, t)-f(1) v(1,3, t)-f(2) v(1,2, t))}{\gamma},
$$

$$
\begin{aligned}
\Theta(i)= & \Re\left\{\left\langle\boldsymbol{s}_{2}, \boldsymbol{s}_{1}\right\rangle f(2 i, \Delta \omega, \Delta \phi)\right\} \\
& -\sum_{t=1}^{T}\left\{\alpha_{1}(t) \alpha_{2}(t)(2 f(0) f(i) \beta(0,1, i, t)\right. \\
& -\beta(0,1,0, t)\left(\beta^{2}(0,1, i, t)-\beta^{2}(1,1, i, t)\right) \\
& \left.\left.-f^{2}(1) \beta(0,1,0, t)-2 \beta(0,1, i, t) \beta(1,1, i, t)\right)\right\} / \gamma,
\end{aligned}
$$

and

$$
\begin{aligned}
\Xi= & \Re\left\{\left\langle\boldsymbol{s}_{2}, \boldsymbol{s}_{1}\right\rangle f(3, \Delta \omega, \Delta \phi)\right\} \\
& -\sum_{t=1}^{T}\left\{\alpha_{1}(t) \alpha_{2}(t)(f(0) f(1) \beta(0,1,2, t)\right. \\
& -\beta(0,1,2, t) v(1,2, t)+f(0) f(2) \beta(0,1,1, t) \\
& -f(1) f(2) \beta(0,1,0, t)+\beta(1,1,2, t) \iota(2,1, t))\} / \gamma .
\end{aligned}
$$

where $f(m, \Delta \omega, \Delta \phi)=\sum_{n=0}^{N-1} d_{n}^{m} \mathrm{e}^{j\left(\Delta \omega d_{n}+\Delta \phi d_{n}^{2}\right)}$ (for sake of clarity $f(i)$ was used instead of $f(i, 0,0))$ in which

$\beta(q, k, l, t)=\Re\left\{j^{q} \mathrm{e}^{j k \Delta \psi(t)} f(l, \Delta \omega, \Delta \phi)\right\}$,

$\iota\left(i_{1}, i_{2}, t\right)=\beta\left(1,1, i_{1}, t\right) \beta\left(0,1, i_{2}, t\right)-\beta\left(1,1, i_{2}, t\right) \beta\left(0,1, i_{1}, t\right)$,

$v\left(i_{1}, i_{2}, t\right)=\beta\left(0,1, i_{1}, t\right) \beta\left(0,1, i_{2}, t\right)+\beta\left(1,1, i_{1}, t\right) \beta\left(1,1, i_{2}, t\right)$,

and

$\gamma=f^{2}(0)-\|f(0, \Delta \omega, \Delta \phi)\|^{2}$.

\section{References}

[1] M.N. El Korso, M. Pesavento, Performance analysis for near field source localization, in: 2012 IEEE 7th Sensor Array and Multichannel Signal Processing Workshop, SAM, IEEE, 2012, pp. 197-200.

[2] J.-P. Delmas, H. Abeida, Stochastic Cramér-Rao bound for noncircular signals with application to DOA estimation, IEEE Trans. Signal Process. 52 (11) (2004) 3192-3199, http://dx.doi.org/10.1109/TSP.2004.836462.

[3] L. Berriche, K. Abed-Meraim, J.-C. Belfiore, Cramér-Rao bounds for MIMO channel estimation, in: IEEE International Conference on Acoustics, Speech, and Signal Processing, Proceedings, vol. 4, 2004, ICASSP '04, 2004, pp. iv397-iv400.

[4] H. Krim, M. Viberg, Two decades of array signal processing research: the parametric approach, IEEE Signal Process. Mag. 13 (4) (1996) 67-94, http://dx.doi.org/10.1109/79.526899.

[5] P. Stoica, A. Nehorai, Performance study of conditional and unconditional direction-of-arrival estimation, IEEE Trans. Acoust. Speech Signal Process. 38 (10) (1990) 1783-1795, http://dx.doi.org/10.1109/29.60109.

[6] Z. Ben-Haim, Y. Eldar, On the constrained Cramér-Rao bound with a singular Fisher information matrix, IEEE Signal Process. Lett. 16 (6) (2009) 453-456, http://dx.doi.org/10.1109/LSP.2009.2016831.

[7] V. Anastasio, A. Farina, F. Colone, P. Lombardo, Cramer-Rao lower bound with pd $<1$ for target localisation accuracy in multistatic passive radar, IET Radar Sonar Navig. 8 (7) (2014) 767-775, http://dx.doi.org/10.1049/iet-rsn.2013.0213. 
[8] A. Noel, K. Cheung, R. Schober, Bounds on distance estimation via diffusive molecular communication, in: 2014 IEEE Global Communications Conference GLOBECOM, 2014, pp. 2813-2819.

[9] R. Boyer, Performance bounds and angular resolution limit for the moving colocated MIMO radar, IEEE Trans. Signal Process. 59 (4) (2011) 1539-1552, http://dx.doi.org/10.1109/TSP.2010.2100387.

[10] F. Foroozan, A. Asif, R. Boyer, Time reversal MIMO radar: improved CRB and angular resolution limit, in: 2013 IEEE International Conference on Acoustics, Speech and Signal Processing, ICASSP, 2013, pp. 4125-4129.

[11] Z. Liu, A. Nehorai, Statistical angular resolution limit for point sources, IEEE Trans. Signal Process. 55 (11) (2007) 5521-5527, http://dx.doi.org/10.1109/ TSP.2007.898789.

[12] D. Lu, Y. Li, C. Liang, Statistical resolution limit based on Cramér-Rao bound, in: IET International Radar Conference 2013, 2013, pp. 1-5.

[13] M.N. El Korso, R. Boyer, A. Renaux, S. Marcos, Conditional and unconditional Cramér-Rao bounds for near-field source localization, IEEE Trans. Signal Process. 58 (5) (2010) 2901-2907, http://dx.doi.org/10.1109/TSP.2010.2043128.

[14] M. Haardt, M. Pesavento, F. Röemer, M.N. El Korso, Subspace methods and exploitation of special array structures, in: M. Viberg (Ed.), Electronic Reference in Signal Processing: Array and Statistical Signal Processing, in: Academic Press Library in Signal Processing, vol. 3, Elsevier Ltd., 2014.

[15] H. Gazzah, J.-P. Delmas, CRB-based design of linear antenna arrays for near-field source localization, IEEE Trans. Antennas Propag. 62 (4) (2014) 1965-1974, http://dx.doi.org/10.1109/TAP.2014.2298882.

[16] E. Grosicki, K. Abed-Meraim, Y. Hua, A weighted linear prediction method for near-field source localization, IEEE Trans. Signal Process. 53 (10) (2005) 3651-3660, http://dx.doi.org/10.1109/TSP.2005.855100.

[17] Y.-D. Huang, M. Barkat, Near-field multiple source localization by passive sensor array, IEEE Trans. Antennas Propag. 39 (7) (1991) 968-975, http://dx.doi.org/10.1109/8.86917.

[18] J.-P. Delmas, Y. Meurisse, On the Cramér-Rao bound and maximum likelihood in passive time delay estimation for complex signals, in: 2012 IEEE International Conference on Acoustics, Speech and Signal Processing, ICASSP, 2012 pp. 3541-3544.

[19] L. Berriche, K. Abed-Meraim, Semi-blind stochastic maximum likelihood for frequency selective MIMO channels, in: IEEE 16th International Symposium on Personal, Indoor and Mobile Radio Communications, vol. 1, 2005, PIMRC 2005, 2005, pp. 552-556.

[20] Y. Begriche, M. Thameri, K. Abed-Meraim, Exact Cramér-Rao bound for near field source localization, in: 2012 11th International Conference on Information Science, Signal Processing and their Applications, ISSPA, 2012, pp. 718-721.

[21] J.-P. Delmas, H. Gazzah, CRB analysis of near-field source localization using uniform circular arrays, in: 2013 IEEE International Conference on Acoustics, Speech and Signal Processing, ICASSP, 2013, pp. 3996-4000.

[22] Y. Begriche, M. Thameri, K. Abed-Meraim, Exact conditional and unconditional Cramér-Rao bounds for near field localization, Digit. Signal Process. 31 (2014) 45-58, http://dx.doi.org/10.1016/j.dsp.2014.04.006.

[23] W. Zhu, J. Tang, S. Wan, Angular resolution limit of two closely-spaced point sources based on hypothesis testing, in: 2013 IEEE International Conference on Acoustics, Speech and Signal Processing, ICASSP, 2013, pp. 3905-3909.

[24] X. Zhang, M.N. El Korso, M. Pesavento, On the asymptotic resolvability of farfield stochastic sources, in: 2012 Proceedings of the 20th European Signal Processing Conference, EUSIPCO, 2012, pp. 889-893.

[25] Y. Meurisse, J.-P. Delmas, Bounds for sparse planar and volume arrays, IEEE Trans. Inf. Theory 47 (1) (2001) 464-468, http://dx.doi.org/10.1109/18.904563.

[26] M.N. El Korso, R. Boyer, A. Renaux, S. Marcos, Statistical resolution limit of the uniform linear cocentered orthogonal loop and dipole array, IEEE Trans. Signa Process. 59 (1) (2011) 425-431, http://dx.doi.org/10.1109/TSP.2010.2083657.

[27] N. Tran, R. Boyer, S. Marcos, P. Larzabal, Angular resolution limit for array processing: estimation and information theory approaches, in: 2012 Proceedings of the 20th European Signal Processing Conference, EUSIPCO, 2012 pp. 101-105.

[28] M. Thameri, R. Boyer, K. Abed-Meraim, Derivation of an analytical expression of the Gaussian model statistical resolution limit, in: 2013 IEEE International Conference on Acoustics, Speech and Signal Processing, ICASSP, 2013, pp. 5529-5533.

[29] M.N. El Korso, R. Boyer, A. Renaux, S. Marcos, Statistical analysis of achievable resolution limit in the near field source localization context, Signal Process. 92 (2) (2012) 547-552.

[30] H. Mir, B. Carlson, Range resolution of unequal strength targets, in: International Radar Conference - Surveillance for a Safer World, 2009, RADAR, 2009, pp. 1-5.
[31] S. Smith, Statistical resolution limits and the complexified Cramér-Rao bound, IEEE Trans. Signal Process. 53 (5) (2005) 1597-1609, http://dx.doi.org/10.1109/ TSP.2005.845426.

[32] H. Cramér, Mathematical Methods of Statistics, Princeton University Press, Princeton, NJ, 1946

[33] C.-Y. Chi, C.-H. Chen, C.-C. Feng, C.-Y. Chen, Fundamentals of statistical signal processing, Blind Equalization and System Identification: Batch Processing Algorithms, Performance and Applications, 2006, pp. 83-182.

[34] P. Stoica, R.L. Moses, Spectral Analysis of Signals, Pearson/Prentice Hall, Upper Saddle River, NJ, 2005.

[35] F.Z. Zhang, The Schur Complement and Its Applications, vol. 4, Springer, 2005.

[36] S.M. Kay, Fundamentals of Statistical Signal Processing: Estimation Theory, Prentice-Hall, Englewood Cliffs, NJ, 1993.

[37] A. D'Amico, M. Morelli, Joint channel and DOA estimation for multicarrier CDMA uplink transmissions, IEEE Trans. Veh. Technol. 58 (1) (2009) 116-125, http://dx.doi.org/10.1109/TVT.2008.921624.

[38] J. Li, R.T. Compton, Maximum likelihood angle estimation for signals with known waveforms, IEEE Trans. Signal Process. 41 (9) (1993) 2850-2862, http://dx.doi.org/10.1109/78.236507.

[39] S. Smith, Accuracy and resolution bounds for adaptive sensor array processing, in: Ninth IEEE SP Workshop on Statistical Signal and Array Processing, Proceedings, 1998, 1998, pp. 37-40.

[40] X. Zhang, M.N. El Korso, M. Pesavento, Angular resolution limit for deterministic correlated sources, in: 2013 IEEE International Conference on Acoustics, Speech and Signal Processing, ICASSP, 2013, pp. 5539-5543. 